\title{
Well-posedness for the microcurl model in both single and polycrystal gradient plasticity
}

\author{
François Ebobisse ${ }^{1}$ and Patrizio Neff ${ }^{2}$ and Samuel Forest ${ }^{3}$
}

May 17, 2022

\begin{abstract}
We consider the recently introduced microcurl model which is a variant of strain gradient plasticity in which the curl of the plastic distortion is coupled to an additional micromorphictype field. For both single crystal and polycrystal cases, we formulate the model and show its well-posedness in the rate-independent case provided some local hardening (isotropic or linear kinematic) is taken into account. To this end, we use the functional analytical framework developed by Han-Reddy. We also compare the model to the relaxed micromorphic model as well as to a dislocation-based gradient plasticity model.
\end{abstract}

Key words: plasticity, gradient plasticity, variational modeling, dissipation function, micromorphic continuum, defect energy, micro-dislocation.

AMS 2010 subject classification: 35D30, 35D35, 74C05, 74C15, 74D10, 35J25.

\footnotetext{
${ }^{1}$ Corresponding author, François Ebobisse, Department of Mathematics and Applied Mathematics, University of Cape Town, Rondebosch 7700, South Africa, e-mail: francois.ebobissebille@uct.ac.za

${ }^{2}$ Patrizio Neff, Lehrstuhl für Nichtlineare Analysis und Modellierung, Fakultät für Mathematik, Universität Duisburg-Essen, Thea-Leymann Str. 9, 45127 Essen, Germany, e-mail: patrizio.neff@uni-due.de, http://www.unidue.de/mathematik/ag_neff

${ }^{3}$ Samuel Forest, MINES ParisTech, Centre des Matériaux, UMR CNRS 7633, BP 87, 91003 Evry, France, e-mail: samuel.forest@ensmp.fr
} 


\section{Contents}

1 Introduction $\quad 2$

2 Some notational agreements and definitions 5

$\begin{array}{lll}3 & \text { The microcurl model in single crystal gradient plasticity } & 7\end{array}$

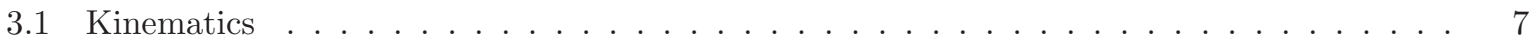

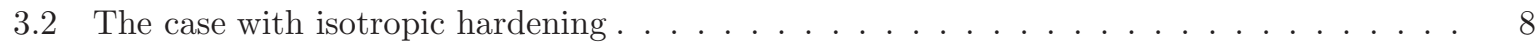

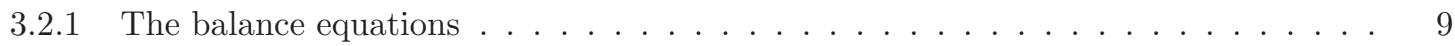

3.2 .2 The derivation of the dissipation inequality. . . . . . . . . . . . . . . 10

3.2 .3 The flow rule . . . . . . . . . . . . . . . . . . . . 11

3.2 .4 Strong formulation of the model . . . . . . . . . . . . . . . . . 12

3.2 .5 Weak formulation of the model . . . . . . . . . . . . . . . . . . . . 12

3.2 .6 Existence result for the weak formulation . . . . . . . . . . . . . . . . . 14

3.2 .7 Uniqueness of the weak/strong solution . . . . . . . . . . . . . . . . 16

3.3 The model with linear kinematical hardening . . . . . . . . . . . . . . . . . . 18

3.3.1 The description of the model . . . . . . . . . . . . . . . . . 18

3.3.2 The weak formulation of the model with linear kinematical hardening . . . . . . 19

3.3.3 Existence and uniqueness for the model with linear kinematic hardening . . . . . . 21

4 The microcurl model in polycrystalline gradient plasticity 23

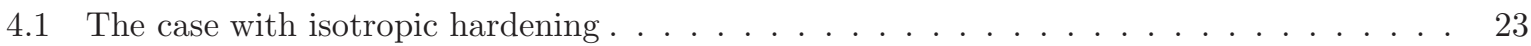

4.1 .1 The balance equations. . . . . . . . . . . . . . . . . . . 23

4.1 .2 The derivation of the dissipation inequality. . . . . . . . . . . . . 23

4.1 .3 The flow rule . . . . . . . . . . . . . . . . . . . . 24

4.1.4 Strong formulation of the model . . . . . . . . . . . . . . . . . 25

4.1.5 Weak formulation of the model . . . . . . . . . . . . . . . . . . 25

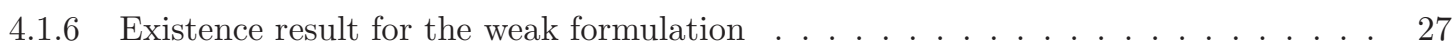

4.2 The model with linear kinematical hardening . . . . . . . . . . . . . . . . . . 29

5 The relaxed linear micromorphic continuum $\quad 30$

6 Conclusion $\quad 31$

\section{Introduction}

In this paper we consider the so-called microcurl model in plasticity. The model was introduced in $[31,17]$ to serve the purpose of augmenting classical plasticity with length scale effects while otherwise keeping the algorithmic structure of classical plasticity. The idea is simple and straight-forward: the in general non-symmetric plastic distortion $p$ (single crystal plasticity and polycrystal plasticity with plastic spin) with its local in space evolution is energetically coupled to a micromorphic-type additional non-symmetric tensorial variable $\mathcal{X}_{p}$ via a penalty-like term $\frac{1}{2} \mu H_{\chi}\left\|p-\mathcal{X}_{p}\right\|^{2}$. The tensor field $\mathcal{X}_{p}$ is generally assumed to be incompatible i.e., it may not derive from a vector field. The total energy in the model is then augmented by a quadratic contribution acting on the Curl of $\mathcal{X}_{p}$. The new variable $\mathcal{X}_{p}$ is now determined by free-variation 
of the energy w.r.t. the displacement field $u$ and the micromorphic field $\mathcal{X}_{p}$ together with corresponding tangential boundary conditions for $\mathcal{X}_{p}$. This generates the usual equilibrium equation on the one hand and what we will call a micro-balance equation for $\mathcal{X}_{p}$ on the other hand.

In the penalty limit $H_{\chi} \rightarrow \infty$, when one expects $p=\mathcal{X}_{p}$, the variable Curl $\mathcal{X}_{p}$ is then interpreted to be the dislocation density tensor Curl $p$. The advantage of such a formulation is clear: there is no need for an extended thermodynamic setting, since $\mathcal{X}_{p}$ is not directly taking part in the dissipation. Constitutive laws including dissipative contributions of the microdeformation and microcurl can be proposed, as done in [34] in the general micromorphic case, but they will require additional material parameters whose identification necessitates material specific physical considerations. Thus, also no higher order boundary conditions at interfaces between elastic and plastic parts need be discussed. The resulting model can therefore be described as a pseudo-regularized strain gradient model.

The microdeformation variable $\mathcal{X}_{p}$ has at least two different interpretations. First, it can be regarded as a mathematical auxiliary variable used to replace the higher order partial differential equations arising in strain gradient plasticity by a system of two sets of second order partial differential equations for the displacement and microdeformation. This method has computational merits for the implementation of strain gradient plasticity models in finite element codes, see [6]. In that case, $H_{\chi}$ is regarded as a mere penalty parameter and should be large enough to enforce the constraint $p=\mathcal{X}_{p}$. In contrast, the microdeformation variable $\mathcal{X}_{p}$ can also be viewed as a constitutive variable with physical interpretation, for instance based on statistical mechanics, $p$ representing the average plastic distortion over the material volume element and $\mathcal{X}_{p}$ being related to the variance of plastic deformation inside this volume. This interpretation is similar to the microconcentration variable introduced in $[92,33]$ to solve Cahn-Hilliard equations. In this context, $H_{\chi}$ must be regarded as a true material higher order modulus to be identified from suitable experimental results. Compared to standard strain gradient plasticity, the microcurl model therefore possesses one additional parameter, $H_{\chi}$, which allows for better description of physical results, as suggested in [18]. An interpretation of the microdeformation $\mathcal{X}_{p}$ was recently proposed in the case of polycrystalline plasticity and damage in $[82,83]$ where it is related to the grain to grain heterogeneitiy of plastic deformation.

Another computational advantage of the microcurl single crystal plasticity model is that the number of independent degrees of freedom ( 9 tensor components of $\mathcal{X}_{p}$, or 8 in the case of incompressible microplasticity) is independent of the crystallographic structure of the material and of the number of slip systems. This is in contrast to strain gradient plasticity models involving the directional gradient of the slip variables [39], which require as many degrees of freedom as slip systems (12 at least in FCC crystals, up to 48 in BCC crystals!). A comparison and discussion of models based on the full dislocation density tensors with models involving densities of geometrically necessary dislocations can be found in [58].

In this paper we will consider the microcurl model in two variants. First, in its original form as a computational approach towards single crystal strain gradient plasticity. We formulate the governing system and show its well-posedness in the rate-independent case. The natural solution space for the micro-variable $\mathcal{X}_{p}$ is the Sobolev-like space $H$ (Curl).

Second, we extend the approach formally to polycrystalline plasticity in which the plastic variable $\varepsilon_{p}:=\operatorname{sym} p$ (the plastic strain) is assumed to be symmetric. In this case we still allow for a non-symmetric micro-variable $\mathcal{X}_{p}$ which is now coupled to the plastic variable only via its 
symmetric part by $\frac{1}{2} \mu H_{\chi}\left\|\operatorname{sym}\left(p-\mathcal{X}_{p}\right)\right\|^{2}$. This represents an alternative to recently proposed strain gradient plasticity models involving a plastic spin tensor for polycrystals [40, 9, 83]. Again, we show the well-posedness of the formulation. Here, we need recently introduced coercive inequalities generalizing Korn's inequality to incompatible tensor fields [72, 73, 74, 75].

The mathematical analysis (with results of existence and uniqueness) for both variants (single crystal and polycrystalline) is obtained through the machinery developped by Han-Reddy [48, Theorems 6.15 and 6.19] for classical plasticity and recently extended to models of gradient plasticity in $[20,68,24,25,22]$. In this approach, the model through the primal form of the flow rule is weakly formulated as a variational inequality and the key issue for its well-posedness is the study of the coercivity of the bilinear form involved on a suitable closed convex subset of some Hilbert space.

The polycrystalline variant of the microcurl model bears some superficial resemblence with the recently introduced relaxed micromorphic models $[69,70]$. For purpose of clarification, we present the relaxed micromorphic model and clearly point out the differences. In order to put the microcurl modelling framework further on display we finish this introduction with another dislocation based strain gradient plasticity model with plastic spin [24, 25, 22], see Table 1 .

\begin{tabular}{|ll|}
\hline Additive split of distortion: & $\nabla u=e+p, \quad \varepsilon^{e}:=\operatorname{sym} e, \quad \varepsilon^{p}:=\operatorname{sym} p$ \\
Equilibrium: & $\operatorname{Div} \sigma+f=0$ with $\sigma=\mathbb{C}_{\mathrm{iso}} \varepsilon^{e}$ \\
Free energy: & $\frac{1}{2}\left\langle\mathbb{C}_{\mathrm{iso}} \varepsilon^{e}, \varepsilon^{e}\right\rangle+\frac{1}{2} \mu k_{1}\left\|\varepsilon^{p}\right\|^{2}+\frac{1}{2} \mu L_{c}^{2}\|\operatorname{Curl} p\|^{2}$ \\
Yield condition: & $\phi\left(\Sigma_{E}\right):=\left\|\operatorname{dev} \Sigma_{E}\right\|-\sigma_{0} \leq 0$ \\
where & $\Sigma_{E}:=\sigma+\Sigma_{\text {curl }}+\Sigma_{\mathrm{kin}}^{\operatorname{lin}}$, \\
& $\Sigma_{\text {curl }}^{\operatorname{lin}}:=-\mu L_{c}^{2} \operatorname{Curl} \operatorname{Curl} p, \quad \Sigma_{\mathrm{kin}}^{\operatorname{lin}}:=-\mu k_{1} \varepsilon^{p}$ \\
& \\
Dissipation inequality: & $\int_{\Omega}\left\langle\Sigma_{E}, \dot{p}\right\rangle d x \geq 0$ \\
Dissipation function: & $\mathcal{D}(q):=\sigma_{0}\|q\|$ \\
Flow rule in primal form: & $\Sigma_{E} \in \partial \mathcal{D}(\dot{p})$ \\
& \\
Flow rule in dual form: & $\dot{p}=\lambda \frac{\operatorname{dev} \Sigma_{E}}{\left\|\operatorname{dev} \Sigma_{E}\right\|}, \quad \lambda=\|\dot{p}\|$ \\
& \\
KKT conditions: & $\lambda \geq 0, \quad \phi\left(\Sigma_{E}\right) \leq 0, \quad \lambda \phi\left(\Sigma_{E}\right)=0$ \\
Boundary conditions for $p:$ & $p \times n=0$ on $\Gamma_{\mathrm{D}}, \quad(\operatorname{Curl} p) \times n=0$ on $\partial \Omega \backslash \Gamma_{\mathrm{D}}$ \\
Function space for $p:$ & $p(t, \cdot) \in \mathrm{H}\left(\operatorname{Curl} ; \Omega, \mathbb{R}^{3 \times 3}\right)$ \\
\hline
\end{tabular}

Table 1: The polycrystalline plasticity model model with linear kinematic hardening and plastic spin studied in $[22]$.

Here, the microcurl-type regularization would be obtained by considering the microcurl energy

$$
\frac{1}{2}\left\langle\mathbb{C}_{\text {iso }} \varepsilon^{e}, \varepsilon^{e}\right\rangle+\frac{1}{2} \mu H_{\chi}\left\|p-\mathcal{X}_{p}\right\|^{2}+\frac{1}{2} \mu k_{1}\|\operatorname{sym} p\|^{2}+\frac{1}{2} \mu L_{c}^{2}\left\|\operatorname{Curl} \mathcal{X}_{p}\right\|^{2}
$$

and for $H_{\chi} \rightarrow \infty$ we would recover the model from Table 1 .

The polycrystalline microcurl variant which we introduce in this paper is, however, based on 
the energy

$$
\frac{1}{2}\left\langle\mathbb{C}_{\text {iso }} \varepsilon^{e}, \varepsilon^{e}\right\rangle+\frac{1}{2} \mu H_{\chi}\left\|\operatorname{sym}\left(p-\mathcal{X}_{p}\right)\right\|^{2}+\frac{1}{2} \mu k_{1}\|\operatorname{sym} p\|^{2}+\frac{1}{2} \mu L_{c}^{2}\left\|\operatorname{Curl} \mathcal{X}_{p}\right\|^{2} .
$$

This ansatz seems to be appropriate for polycrystalline plasticity without plastic spin.

\section{Some notational agreements and definitions}

Let $\Omega$ be a bounded domain in $\mathbb{R}^{3}$ with Lipschitz continuous boundary $\partial \Omega$, which is occupied by the elastoplastic body in its undeformed configuration. Let $\Gamma_{\mathrm{D}}$ be a smooth subset of $\partial \Omega$ with non-vanishing 2-dimensional Hausdorff measure. A material point in $\Omega$ is denoted by $x$ and the time domain under consideration is the interval $[0, T]$.

For every $a, b \in \mathbb{R}^{3}$, we let $\langle a, b\rangle_{\mathbb{R}^{3}}$ denote the scalar product on $\mathbb{R}^{3}$ with associated vector norm $|a|_{\mathbb{R}^{3}}^{2}=\langle a, a\rangle_{\mathbb{R}^{3}}$. We denote by $\mathbb{R}^{3 \times 3}$ the set of real $3 \times 3$ tensors. The standard Euclidean scalar product on $\mathbb{R}^{3 \times 3}$ is given by $\langle A, B\rangle_{\mathbb{R}^{3 \times 3}}=\operatorname{tr}\left[A B^{T}\right]$, where $B^{T}$ denotes the transpose tensor of $B$. Thus, the Frobenius tensor norm is $\|A\|^{2}=\langle A, A\rangle_{\mathbb{R}^{3 \times 3}}$. In the following we omit the subscripts $\mathbb{R}^{3}$ and $\mathbb{R}^{3 \times 3}$. The identity tensor on $\mathbb{R}^{3 \times 3}$ will be denoted by $\mathbb{1}$, so that $\operatorname{tr}(A)=$ $\langle A, \mathbb{1}\rangle$. The set $\mathfrak{s o}(3):=\left\{X \in \mathbb{R}^{3 \times 3} \mid X^{T}=-X\right\}$ is the Lie-Algebra of skew-symmetric tensors. We let $\operatorname{Sym}(3):=\left\{X \in \mathbb{R}^{3 \times 3} \mid X^{T}=X\right\}$ denote the vector space of symmetric tensors and $\mathfrak{s l}(3):=\left\{X \in \mathbb{R}^{3 \times 3} \mid \operatorname{tr}(X)=0\right\}$ be the Lie-Algebra of traceless tensors. For every $X \in \mathbb{R}^{3 \times 3}$, we set $\operatorname{sym}(X)=\frac{1}{2}\left(X+X^{T}\right)$, skew $(X)=\frac{1}{2}\left(X-X^{T}\right)$ and $\operatorname{dev}(X)=X-\frac{1}{3} \operatorname{tr}(X) \mathbb{1} \in \mathfrak{s l}(3)$ for the symmetric part, the skew-symmetric part and the deviatoric part of $X$, respectively. Quantities which are constant in space will be denoted with an overbar, e.g., $\bar{A} \in \mathfrak{s o}(3)$ for the function $A: \mathbb{R}^{3} \rightarrow \mathfrak{s o}(3)$ which is constant with constant value $\bar{A}$.

The body is assumed to undergo infinitesimal deformations. Its behaviour is governed by a set of equations and constitutive relations. Below is a list of variables and parameters used throughout the paper with their significations:

- $u$ is the displacement of the macroscopic material points;

- $p$ is the infinitesimal plastic distortion variable which is a non-symmetric second order tensor, incapable of sustaining volumetric changes; that is, $p \in \mathfrak{s l}(3)$. The tensor $p$ represents the average plastic slip; $p$ is not a state-variable, while the rate $\dot{p}$ is an infinitesimal state variable in some suitable sense;

- $e=\nabla u-p$ is the infinitesimal elastic distortion which is in general a non-symmetric second order tensor and is an infinitesimal state-variable;

- $\varepsilon_{p}=\operatorname{sym} p$ is the symmetric infinitesimal plastic strain tensor, which is trace free, $\varepsilon_{p} \in$ $\mathfrak{s l}(3) ; \varepsilon_{p}$ is not a state-variable; the rate $\dot{\varepsilon}_{p}$ is an infinitesimal state-variable;

- $\varepsilon_{e}=\operatorname{sym} \nabla u-\varepsilon_{p}$ is the symmetric infinitesimal elastic strain tensor and is an infinitesimal state-variable;

- $\mathcal{X}_{p} \in \mathbb{R}^{3 \times 3}$ is the non-symmetric infinitesimal micro-distortion with sym $\mathcal{X}_{p}$ being the symmetric micro-strain; 
- $\sigma$ is the Cauchy stress tensor which is a symmetric second order tensor and is an infinitesimal state-variable;

- $\sigma_{0}$ is the initial yield stress for plastic variables $p$ or $\varepsilon_{p}:=\operatorname{sym} p$ and is an infinitesimal state-variable;

- $f$ is the body force;

- $\operatorname{Curl} p=\alpha$ is the dislocation density tensor satisfying the so-called Bianchi identities $\operatorname{Div} \alpha=0$ and is an infinitesimal state-variable;

- $\eta_{p}=\int_{0}^{t}\left\|\dot{\varepsilon}_{p}\right\| d s$ is the accumulated equivalent plastic strain and is an infinitesimal statevariable;

- $\gamma^{\alpha}$ is the slip in the $\alpha$-th slip system in single crystal plasticity while $l^{\alpha}$ is the slip direction and $\nu^{\alpha}$ is the normal vector to the slip plane with $\alpha=1, \ldots, n_{\text {slip }}$. Hence, $p=\sum_{\alpha} \gamma^{\alpha} l^{\alpha} \otimes \nu^{\alpha}$.

For isotropic media, the fourth order isotropic elasticity tensor $\mathbb{C}_{\text {iso }}: \operatorname{Sym}(3) \rightarrow \operatorname{Sym}(3)$ is given by

$$
\mathbb{C}_{\text {iso }} \operatorname{sym} X=2 \mu \text { dev } \operatorname{sym} X+\kappa \operatorname{tr}(X) \mathbb{1}=2 \mu \operatorname{sym} X+\lambda \operatorname{tr}(X) \mathbb{1}
$$

for any second-order tensor $X$, where $\mu$ and $\lambda$ are the Lamé moduli satisfying

$$
\mu>0 \text { and } 3 \lambda+2 \mu>0
$$

and $\kappa>0$ is the bulk modulus. These conditions suffice for pointwise positive definiteness of the elasticity tensor in the sense that there exists a constant $m_{0}>0$ such that

$$
\forall X \in \mathbb{R}^{3 \times 3}: \quad\left\langle\operatorname{sym} X, \mathbb{C}_{\text {iso }} \operatorname{sym} X\right\rangle \geq m_{0}\|\operatorname{sym} X\|^{2} .
$$

The space of square integrable functions is $L^{2}(\Omega)$, while the Sobolev spaces used in this paper are:

$$
\begin{aligned}
\mathrm{H}^{1}(\Omega)= & \left\{u \in L^{2}(\Omega) \mid \operatorname{grad} u \in L^{2}(\Omega)\right\}, \quad \operatorname{grad}=\nabla, \\
& \|u\|_{H^{1}(\Omega)}^{2}=\|u\|_{L^{2}(\Omega)}^{2}+\|\operatorname{grad} u\|_{L^{2}(\Omega)}^{2}, \quad \forall u \in \mathrm{H}^{1}(\Omega), \\
\mathrm{H}(\operatorname{curl} ; \Omega)= & \left\{v \in L^{2}(\Omega) \mid \operatorname{curl} v \in L^{2}(\Omega)\right\}, \quad \operatorname{curl}=\nabla \times, \\
& \|v\|_{\mathrm{H}(\operatorname{curl} ; \Omega)}^{2}=\|v\|_{L^{2}(\Omega)}^{2}+\|\operatorname{curl} v\|_{L^{2}(\Omega)}^{2}, \quad \forall v \in \mathrm{H}(\operatorname{curl} ; \Omega) .
\end{aligned}
$$

For every $X \in C^{1}\left(\Omega, \mathbb{R}^{3 \times 3}\right)$ with rows $X^{1}, X^{2}, X^{3}$, we use in this paper the definition of Curl $X$ in $[68,90]$ :

$$
\operatorname{Curl} X=\left(\begin{array}{l}
\operatorname{curl} X^{1}-- \\
\operatorname{curl} X^{2}-- \\
\operatorname{curl} X^{3}--
\end{array}\right) \in \mathbb{R}^{3 \times 3},
$$

for which Curl $\nabla v=0$ for every $v \in C^{2}\left(\Omega, \mathbb{R}^{3}\right)$. Notice that the definition of Curl $X$ above is such that $(\operatorname{Curl} X)^{T} a=\operatorname{curl}\left(X^{T} a\right)$ for every $a \in \mathbb{R}^{3}$ and this clearly corresponds to the 
transpose of the Curl of a tensor as defined in [41, 43].

The following function spaces and norms will also be used later.

$$
\begin{aligned}
\mathrm{H}\left(\mathrm{Curl} ; \Omega, \mathbb{R}^{3 \times 3}\right) & =\left\{X \in L^{2}\left(\Omega, \mathbb{R}^{3 \times 3}\right) \mid \operatorname{Curl} X \in L^{2}\left(\Omega, \mathbb{R}^{3 \times 3}\right)\right\}, \\
\|X\|_{\mathrm{H}(\mathrm{Curl} ; \Omega)}^{2} & =\|X\|_{L^{2}(\Omega)}^{2}+\|\operatorname{Curl} X\|_{L^{2}(\Omega)}^{2}, \quad \forall X \in \mathrm{H}\left(\operatorname{Curl} ; \Omega, \mathbb{R}^{3 \times 3}\right), \\
\mathrm{H}(\operatorname{Curl} ; \Omega, \mathbb{E}) & =\left\{X: \Omega \rightarrow \mathbb{E} \mid X \in \mathrm{H}\left(\operatorname{Curl} ; \Omega, \mathbb{R}^{3 \times 3}\right)\right\},
\end{aligned}
$$

for $\mathbb{E}:=\mathfrak{s l}(3)$ or $\operatorname{Sym}(3) \cap \mathfrak{s l}(3)$.

We also consider the space

$$
\mathrm{H}_{0}\left(\mathrm{Curl} ; \Omega, \Gamma_{\mathrm{D}}, \mathbb{R}^{3 \times 3}\right)
$$

as the completion in the norm in (2.6) of the space $\left\{X \in C^{\infty}\left(\Omega, \mathbb{R}^{3 \times 3}\right)|X \times n|_{\Gamma_{\mathrm{D}}}=0\right\}$. Therefore, this space generalizes the tangential Dirichlet boundary condition

$$
X \times\left. n\right|_{\Gamma_{\mathrm{D}}}=0
$$

to be satisfied by the plastic micro-distortion $\mathcal{X}_{p}$. The space

$$
\mathrm{H}_{0}\left(\operatorname{Curl} ; \Omega, \Gamma_{\mathrm{D}}, \mathbb{E}\right)
$$

is defined as in (2.6).

The divergence operator Div on second order tensor-valued functions is also defined row-wise as

$$
\operatorname{Div} X=\left(\begin{array}{c}
\operatorname{div} X_{1} \\
\operatorname{div} X_{2} \\
\operatorname{div} X_{3}
\end{array}\right)
$$

\section{The microcurl model in single crystal gradient plasticity}

\subsection{Kinematics}

Single-crystal plasticity is based on the assumption that the plastic deformation happens through crystallograpic shearing which represents the dislocation motion along specific slip systems, each being characterized by a plane with unit normal $\nu^{\alpha}$ and slip direction $l^{\alpha}$ on that plane, and slips $\gamma^{\alpha}\left(\alpha=1, \ldots, n_{\text {slip }}\right)$. The flow rule for the plastic distortion $p$ is written at the slip system level by means of the orientation tensor $m^{\alpha}$ defined as

$$
m^{\alpha}:=l^{\alpha} \otimes \nu^{\alpha}
$$

Under these conditions the plastic distortion $p$ takes the form

$$
p=\sum_{\alpha=1}^{n_{\text {slip }}} \gamma^{\alpha} m^{\alpha}
$$


so that the plastic strain $\varepsilon_{p}=\operatorname{sym} p$ is

$$
\varepsilon_{p}=\sum_{\alpha=1}^{n_{\text {slip }}} \gamma^{\alpha} \operatorname{sym}\left(m^{\alpha}\right)=\frac{1}{2} \sum_{\alpha=1}^{n_{\text {slip }}} \gamma^{\alpha}\left(l^{\alpha} \otimes \nu^{\alpha}+\nu^{\alpha} \otimes l^{\alpha}\right)
$$

and $\operatorname{tr}(p)=0$ since $l^{\alpha} \perp \nu^{\alpha}$.

For the slips $\gamma^{\alpha}\left(\alpha=1, \ldots, n_{\text {slip }}\right)$ we set

$$
\underline{\gamma}:=\left(\gamma^{1}, \ldots, \gamma^{n_{\text {slip }}}\right)
$$

Therefore, we get from (3.3) that

$$
p=\bar{m} \underline{\gamma},
$$

where $\bar{m}$ is the third order tensor ${ }^{1}$ defined as

$$
\bar{m}_{i j \alpha}:=m_{i j}^{\alpha}=l_{i}^{\alpha} \nu_{j}^{\alpha} \quad \text { for } \quad i, j=1,2,3 \text { and } \alpha=1, \ldots, n_{\text {slip }} .
$$

Let $\underline{\eta}:=\left(\eta^{1}, \ldots, \eta^{n_{\text {slip }}}\right)$ with $\eta^{\alpha}$ being a hardening variable in the $\alpha$-th slip system.

\subsection{The case with isotropic hardening}

The starting point is the total energy

$$
\mathcal{E}\left(u, \underline{\gamma}, \mathcal{X}_{p}, \underline{\eta}\right):=\int_{\Omega}\left[\Psi\left(\nabla u, \underline{\gamma}, \mathcal{X}_{p}, \operatorname{Curl} \mathcal{X}_{p}, \underline{\eta}\right)-\langle f, u\rangle\right] d x
$$

where the free-energy density $\Psi$ is given in the additively separated form

$$
\begin{aligned}
\Psi\left(\nabla u, \underline{\gamma}, \mathcal{X}_{p}, \operatorname{Curl} \mathcal{X}_{p}, \underline{\eta}\right):= & \underbrace{\Psi_{e}^{\operatorname{lin}}\left(\varepsilon_{e}\right)}_{\text {elastic energy }}+\underbrace{\Psi_{\text {micro }}^{\operatorname{lin}}\left(p, \mathcal{X}_{p}\right)}_{\text {micro energy }} \\
& +\underbrace{\Psi_{\text {curl }}^{\text {lin }\left(\operatorname{Curl} \mathcal{X}_{p}\right)}}_{\text {defect-like energy }(\mathrm{GND})}+\underbrace{\Psi_{\text {iso }}(\underline{\eta})}_{\text {hardening energy }}
\end{aligned},
$$

where

$$
\begin{aligned}
\Psi_{e}^{\operatorname{lin}}\left(\varepsilon_{e}\right) & :=\frac{1}{2}\left\langle\varepsilon_{e}, \mathbb{C}_{\text {iso }} \varepsilon_{e}\right\rangle=\frac{1}{2}\left\langle\operatorname{sym}(\nabla u-p), \mathbb{C}_{\text {iso }} \operatorname{sym}(\nabla u-p)\right\rangle, \\
\Psi_{\text {micro }}^{\operatorname{lin}}\left(p, \mathcal{X}_{p}\right) & :=\frac{1}{2} \mu H_{\chi}\left\|p-\mathcal{X}_{p}\right\|^{2}, \quad \Psi_{\text {curl }}^{\operatorname{lin}}\left(\operatorname{Curl} \mathcal{X}_{p}\right):=\frac{1}{2} \mu L_{c}^{2}\left\|\operatorname{Curl} \mathcal{X}_{p}\right\|^{2}, \\
\Psi_{\text {iso }}(\underline{\eta}) & :=\frac{1}{2} \mu k_{2}\|\underline{\eta}\|^{2}=\frac{1}{2} \mu k_{2} \sum_{\alpha}\left|\eta^{\alpha}\right|^{2} .
\end{aligned}
$$

Here, $L_{c} \geq 0$ is an energetic length scale which characterizes the contribution of the defectlike energy density to the system, $H_{\chi}$ is a positive nondimensional penalty constant, $k_{2}$ is a positive nondimensional isotropic hardening constant.

\footnotetext{
${ }^{1}$ The terminology "tensor" used here is just intended as "matrix" since $\bar{m}$ does not fulfill the rules of change of orthogonal bases for the last index and therefore is not a tensor in the usual sense.
} 
The starting point for the derivation of the equations and inequalities describing the plasticity model is the two-field minimization formulation

$$
\mathcal{E}\left(u, \underline{\gamma}, \mathcal{X}_{p}, \underline{\eta}\right) \rightarrow \text { min. w.r.t. }\left(u, \mathcal{X}_{p}\right) .
$$

The first variations of the total energy w.r.t. to the variables $u$ and $\mathcal{X}_{p}$ lead to the balance equations in the next section.

\subsubsection{The balance equations}

The conventional macroscopic force balance leads to the equation of equilibrium

$$
\operatorname{div} \sigma+f=0
$$

in which $\sigma$ is the infinitesimal symmetric Cauchy stress and $f$ is the body force.

An additional microscopic balance equation is obtained as follows. Precisely, the first variation of the total energy w.r.t $\mathcal{X}_{p}$ gives for every $\delta \mathcal{X}_{p} \in C^{\infty}\left(\Omega, \mathbb{R}^{3 \times 3}\right)$,

$$
\begin{aligned}
0 & =\left.\frac{d}{d t} \mathcal{E}\left(u, p, \mathcal{X}_{p}+t \delta \mathcal{X}_{p}, \underline{\eta}\right)\right|_{t=0} \\
= & \int_{\Omega}\left[\mu H_{\chi}\left\langle\mathcal{X}_{p}-p, \delta \mathcal{X}_{p}\right\rangle+\mu L_{c}^{2},\left\langle\operatorname{Curl} \mathcal{X}_{p}, \operatorname{Curl} \delta \mathcal{X}_{p}\right\rangle\right] d x \\
= & \int_{\Omega}\left[\mu H_{\chi}\left\langle\mathcal{X}_{p}-p, \delta \mathcal{X}_{p}\right\rangle+\mu L_{c}^{2},\left\langle\operatorname{Curl} \operatorname{Curl} \mathcal{X}_{p}, \delta \mathcal{X}_{p}\right\rangle\right. \\
& \left.+\sum_{i=1}^{3} \operatorname{div}\left(\mu L_{c}^{2} \delta \mathcal{X}_{p}^{i} \times\left(\operatorname{Curl} \mathcal{X}_{p}\right)^{i}\right)\right] d x \\
= & \int_{\Omega}\left\langle\mu H_{\chi}\left(\mathcal{X}_{p}-p\right)+\mu L_{c}^{2}\left\langle\operatorname{Curl} \operatorname{Curl} \mathcal{X}_{p}, \delta \mathcal{X}_{p}\right\rangle\right. \\
& +\sum_{i=1}^{3} \int_{\partial \Omega} \mu L_{c}^{2}\left\langle\delta \mathcal{X}_{p}^{i} \times\left(\operatorname{Curl} \mathcal{X}_{p}\right)^{i}, n\right\rangle d a
\end{aligned}
$$

which on the one hand gives from the choice $\delta \mathcal{X}_{p} \in C_{c}^{\infty}\left(\Omega, \mathbb{R}^{3 \times 3}\right)$ the micro-balance in strong formulation $^{2}$

$$
\mu L_{c}^{2} \operatorname{Curl} \operatorname{Curl} \mathcal{X}_{p}=-\mu H_{\chi}\left(\mathcal{X}_{p}-p\right) .
$$

One the other hand we get

$$
\sum_{i=1}^{3} \int_{\partial \Omega} \mu L_{c}^{2}\left\langle\delta \mathcal{X}_{p}^{i} \times\left(\operatorname{Curl} \mathcal{X}_{p}\right)^{i}, n\right\rangle d a=0 \quad \forall \delta \mathcal{X}_{p} \in C^{\infty}\left(\Omega, \mathbb{R}^{3 \times 3}\right)
$$

which is satisfied if we choose certain homogeneous boundary conditions on the micro-distortion $\mathcal{X}_{p}$. Following Gurtin [40] and also Gurtin and Needleman [44] we choose the simple boundary condition

$$
\mathcal{X}_{p} \times\left. n\right|_{\Gamma_{\mathrm{D}}}=0 \quad \text { and } \quad\left(\operatorname{Curl} \mathcal{X}_{p}\right) \times\left. n\right|_{\partial \Omega \backslash \Gamma_{\mathrm{D}}}=0
$$

\footnotetext{
${ }^{2}$ Here, we have assumed uniform material constants for simplicity.
} 
which in the case of models in strain gradient plasticity, where $\mathcal{X}_{p}$ is replaced by $p$ or $\varepsilon_{p}$ simply implies that there is no flow of the plastic distortion or plastic strain across the piece $\Gamma$ of the boundary $\partial \Omega$.

\subsubsection{The derivation of the dissipation inequality.}

The local free-energy imbalance states that

$$
\dot{\Psi}-\left\langle\sigma, \dot{\varepsilon}_{e}\right\rangle-\langle\sigma, \dot{p}\rangle \leq 0 \text {. }
$$

Now we expand the first term, substitute (3.7)-(3.8) and get

$$
\left\langle\mathbb{C}_{\text {iso }} \varepsilon_{e}-\sigma, \dot{\varepsilon}_{e}\right\rangle-\sum_{\alpha}\left\langle\sigma, m^{\alpha}\right\rangle \dot{\gamma}^{\alpha}+\sum_{\alpha} \frac{\partial \Psi_{\text {micro }}^{\text {lin }}}{\partial \gamma^{\alpha}} \dot{\gamma}^{\alpha}+\sum_{\alpha} \frac{\partial \Psi_{\text {iso }}}{\partial \eta^{\alpha}} \dot{\eta}^{\alpha} \leq 0 .
$$

That is

$$
\left\langle\mathbb{C}_{\mathrm{iso}} \varepsilon_{e}-\sigma, \dot{\varepsilon}_{e}\right\rangle-\sum_{\alpha}\left\langle\sigma, m^{\alpha}\right\rangle \dot{\gamma}^{\alpha}-\sum_{\alpha} \mu H_{\chi}\left\langle\mathcal{X}_{p}-p, m^{\alpha}\right\rangle \dot{\gamma}^{\alpha}+\mu k_{2} \sum_{\alpha} \eta^{\alpha} \dot{\eta}^{\alpha} \leq 0 .
$$

Therefore we obtain

$$
0 \leq-\left\langle\mathbb{C}_{\mathrm{iso}} \varepsilon_{e}-\sigma, \dot{\varepsilon}_{e}\right\rangle+\sum_{\alpha}\left[\left(\tau^{\alpha}+s^{\alpha}\right) \dot{\gamma}^{\alpha}+g^{\alpha} \dot{\eta}^{\alpha}\right]=-\left\langle\mathbb{C}_{\mathrm{iso}} \varepsilon_{e}-\sigma, \dot{\varepsilon}_{e}\right\rangle+\sum_{\alpha}\left[\tau_{\mathrm{E}}^{\alpha} \dot{\gamma}^{\alpha}+g^{\alpha} \dot{\eta}^{\alpha}\right]
$$

where we set

$$
\begin{aligned}
\tau^{\alpha} & :=\left\langle\sigma, m^{\alpha}\right\rangle \quad(\text { resolved shear stress for the } \alpha \text {-th slip system) } \\
s^{\alpha} & :=\mu H_{\chi}\left\langle\mathcal{X}_{p}-p, m^{\alpha}\right\rangle=-\mu L_{c}^{2}\left\langle\text { Curl Curl } \mathcal{X}_{p}, m^{\alpha}\right\rangle, \\
g^{\alpha} & \left.:=-\mu k_{2} \eta^{\alpha} \quad \text { (thermodynamic force power-conjugate to } \dot{\eta}^{\alpha}\right), \\
\tau_{\mathrm{E}}^{\alpha} & :=\tau^{\alpha}+s^{\alpha}=\left\langle\Sigma_{E}, m^{\alpha}\right\rangle,
\end{aligned}
$$

with $\Sigma_{E}$ being the non-symmetric Eshelby-type stress tensor defined by

$$
\Sigma_{E}:=\sigma+\mu H_{\chi}\left(\mathcal{X}_{p}-p\right)=\sigma-\mu L_{c}^{2} \operatorname{Curl} \operatorname{Curl} \mathcal{X}_{p} .
$$

Since the inequality (3.18) must be satisfied for whatever elastic-plastic deformation mechanism, inlcuding purely elastic ones (for which $\dot{\gamma}^{\alpha}=0, \dot{\eta}^{\alpha}=0$ ), equation (3.18) implies the usual infinitesimal elastic stress-strain relation

$$
\begin{aligned}
\sigma=\mathbb{C}_{\text {iso }} \varepsilon_{e} & =2 \mu \operatorname{sym}(\nabla u-p)+\lambda \operatorname{tr}(\nabla u-p) \mathbb{1} \\
& =2 \mu\left(\operatorname{sym}(\nabla u)-\varepsilon_{p}\right)+\lambda \operatorname{tr}(\nabla u) \mathbb{1}
\end{aligned}
$$

and the local reduced dissipation inequality

$$
\sum_{\alpha}\left[\tau_{E}^{\alpha} \dot{\gamma}^{\alpha}+g^{\alpha} \dot{\eta}^{\alpha}\right] \geq 0
$$

which can also be written in compact form as

$$
\sum_{\alpha}\left\langle\Sigma_{p}^{\alpha}, \dot{\eta}_{p}^{\alpha}\right\rangle \geq 0
$$

where we define

$$
\Sigma_{p}^{\alpha}:=\left(\tau_{E}^{\alpha}, g^{\alpha}\right) \quad \text { and } \quad \Gamma_{p}^{\alpha}:=\left(\gamma^{\alpha}, \eta^{\alpha}\right)
$$




\subsubsection{The flow rule}

We consider a yield function on the $\alpha$-th slip system defined by

$$
\phi\left(\Sigma_{p}^{\alpha}\right):=\left|\tau_{\mathrm{E}}^{\alpha}\right|+g^{\alpha}-\sigma_{0} \quad \text { for } \quad \Sigma_{p}^{\alpha}=\left(\tau_{\mathrm{E}}^{\alpha}, g^{\alpha}\right) .
$$

Here, $\sigma_{0}$ is the yield stress of the material, that we assume to be constant on all slip systems and therefore, $\sigma_{\mathrm{y}}^{\alpha}:=\sigma_{0}-g^{\alpha}$ represents the current yield stress for the $\alpha$-th slip system ${ }^{3}$. So the set of admissible generalized stresses for the $\alpha$-th slip system is defined as

$$
\mathcal{K}^{\alpha}:=\left\{\Sigma_{p}^{\alpha}=\left(\tau_{\mathrm{E}}^{\alpha}, g^{\alpha}\right) \mid \phi\left(\Sigma_{p}^{\alpha}\right) \leq 0, g^{\alpha} \leq 0\right\},
$$

with its interior $\operatorname{Int}\left(\mathcal{K}^{\alpha}\right)$ and its boundary $\partial \mathcal{K}^{\alpha}$ being the generalized elastic region and the yield surface for the $\alpha$-th slip system, respectively.

The principle of maximum dissipation ${ }^{4}$ associated with the $\alpha$-th slip system gives us the normality law

$$
\dot{\Gamma}_{p}^{\alpha} \in N_{\mathcal{K}^{\alpha}}\left(\Sigma_{p}^{\alpha}\right)
$$

where $N_{\mathcal{K}^{\alpha}}\left(\Sigma_{p}^{\alpha}\right)$ denotes the normal cone to $\mathcal{K}^{\alpha}$ at $\Sigma_{p}^{\alpha}$. That is, $\dot{\Gamma}_{p}^{\alpha}$ satisfies

$$
\left\langle\bar{\Sigma}^{\alpha}-\Sigma_{p}^{\alpha}, \dot{\Gamma}_{p}^{\alpha}\right\rangle \leq 0 \quad \text { for all } \bar{\Sigma}^{\alpha} \in \mathcal{K}^{\alpha} .
$$

Notice that $N_{\mathcal{K}^{\alpha}}=\partial \chi_{\mathcal{K}^{\alpha}}$, where $\chi_{\mathcal{K}^{\alpha}}$ denotes the indicator function of the set $\mathcal{K}^{\alpha}$ and $\partial \chi_{\mathcal{K}^{\alpha}}$ denotes the subdifferential of the function $\chi_{\mathcal{K}^{\alpha}}$.

Whenever the yield surface $\partial \mathcal{K}^{\alpha}$ is smooth at $\Sigma_{p}^{\alpha}$ then

$$
\dot{\Gamma}_{p}^{\alpha} \in N_{\mathcal{K}^{\alpha}}\left(\Sigma_{p}^{\alpha}\right) \Rightarrow \exists \lambda^{\alpha} \text { such that } \dot{\gamma}^{\alpha}=\lambda^{\alpha} \frac{\tau_{\mathrm{E}}^{\alpha}}{\left|\tau_{\mathrm{E}}^{\alpha}\right|} \quad \text { and } \quad \dot{\eta}^{\alpha}=\lambda^{\alpha}=\left|\dot{\gamma}^{\alpha}\right|
$$

with the Karush-Kuhn Tucker conditions: $\lambda^{\alpha} \geq 0, \phi\left(\Sigma_{p}^{\alpha}\right) \leq 0$ and $\lambda^{\alpha} \phi\left(\Sigma_{p}^{\alpha}\right)=0$. Using convex analysis (Legendre-transformation) we find that

$$
\underbrace{\dot{\Gamma}_{p}^{\alpha} \in \partial \chi_{\mathcal{K}^{\alpha}\left(\Sigma_{p}^{\alpha}\right)}^{\alpha}}
$$

flow rule in its dual formulation for the $\alpha$-th slip system

$$
\underbrace{\Uparrow} \underbrace{\Sigma_{p}^{\alpha} \in \partial \chi_{\mathcal{K}^{\alpha}}^{*}\left(\dot{\Gamma}_{p}^{\alpha}\right)}
$$

\section{flow rule in its primal formulation for the $\alpha$-th slip system}

\footnotetext{
${ }^{3}$ Note that, for the sake of simplicity, the presented isotropic hardening rule $g^{\alpha}$ does not involve latent hardening and the associated interaction matrix, see [36] for a discussion on uniqueness in the presence of latent hardening.

${ }^{4}$ The principle of maximum dissipation (PMD) is shown to be closely related to the so-called minimum principle for the dissipation potential (MPDP) [47, 46, 81], which states that the rate of the internal variables is the minimizer of a functional consisting of the sum of the rate of the free energy and the dissipation function with respect to appropriate boundary conditions. Notice that, as pointed out in [22], both PMD and MPDP are not physical principles but thermodynamically consistent selection rules which turn out to be convenient if no other information is available or if existing flow rules are to be extended to a more general situation.
} 
where $\chi_{\mathcal{K}^{\alpha}}^{*}$ is the Fenchel-Legendre dual of the function $\chi_{\mathcal{K}^{\alpha}}$ denoted in this context by $\mathcal{D}_{\text {iso }}^{\alpha}$, the one-homogeneous dissipation function for the $\alpha$-th slip system. That is, for every $\Gamma^{\alpha}=\left(q^{\alpha}, \beta^{\alpha}\right)$,

$$
\begin{aligned}
\mathcal{D}_{\text {iso }}^{\alpha}\left(\Gamma^{\alpha}\right) & =\sup \left\{\left\langle\Sigma_{p}^{\alpha}, \Gamma^{\alpha}\right\rangle \mid \Sigma_{p}^{\alpha} \in \mathcal{K}^{\alpha}\right\} \\
& =\sup \left\{\tau_{E}^{\alpha} q^{\alpha}+g^{\alpha} \beta^{\alpha} \mid \phi\left(\Sigma_{E}^{\alpha}, g^{\alpha}\right) \leq 0, g^{\alpha} \leq 0\right\} \\
& = \begin{cases}\sigma_{0}\left|q^{\alpha}\right| & \text { if }\left|q^{\alpha}\right| \leq \beta^{\alpha}, \\
\infty & \text { otherwise. }\end{cases}
\end{aligned}
$$

We get from the definition of the subdifferential $\left(\Sigma_{p}^{\alpha} \in \partial \chi_{\mathcal{K}^{\alpha}}^{*}\left(\dot{\Gamma}_{p}^{\alpha}\right)\right)$ that

$$
\mathcal{D}_{\text {iso }}^{\alpha}\left(\Gamma^{\alpha}\right) \geq \mathcal{D}_{\text {iso }}^{\alpha}\left(\dot{\Gamma}_{p}^{\alpha}\right)+\left\langle\Sigma_{p}^{\alpha}, \Gamma^{\alpha}-\dot{\Gamma}_{p}^{\alpha}\right\rangle \text { for any } \Gamma^{\alpha}
$$

That is,

$$
\mathcal{D}_{\text {iso }}^{\alpha}\left(q^{\alpha}, \beta^{\alpha}\right) \geq \mathcal{D}_{\text {iso }}^{\alpha}\left(\dot{\gamma}^{\alpha}, \dot{\eta}^{\alpha}\right)+\tau_{\mathrm{E}}^{\alpha}\left(q^{\alpha}-\dot{\gamma}^{\alpha}\right)+g^{\alpha}\left(\beta^{\alpha}-\dot{\eta}^{\alpha}\right) \quad \text { for any }\left(q^{\alpha}, \beta^{\alpha}\right) .
$$

In the next sections, we present a complete mathematical analysis of the model including both strong and weak formulations as well as a corresponding existence result.

\subsubsection{Strong formulation of the model}

To summarize, we have obtained the following strong formulation for the microcurl model in the single crystal infinitesimal gradient plasticity case with isotropic hardening. Given $f \in$ $\mathrm{H}^{1}\left(0, T ; L^{2}\left(\Omega, \mathbb{R}^{3}\right)\right)$, the goal is to find:

(i) the displacement $u \in \mathrm{H}^{1}\left(0, T ; H_{0}^{1}\left(\Omega, \Gamma_{\mathrm{D}}, \mathbb{R}^{3}\right)\right)$,

(ii) the infinitesimal plastic slips $\gamma^{\alpha} \in \mathrm{H}^{1}\left(0, T ; L^{2}(\Omega)\right)$ for $\alpha=1, \ldots, n_{\text {slip }}$, the infinitesimal micro-distortion $\mathcal{X}_{p} \in \mathrm{H}^{1}\left(0, T ; H\left(\mathrm{Curl} ; \Omega, \mathbb{R}^{3 \times 3}\right)\right.$, with Curl Curl $\mathcal{X}_{p} \in \mathrm{H}^{1}\left(0, T ; L^{2}\left(\Omega, \mathbb{R}^{3 \times 3}\right)\right)$

such that the content of Table 2 holds.

\subsubsection{Weak formulation of the model}

Assume that the problem in Section 3.2.4 has a solution $\left(u, \underline{\gamma}, \mathcal{X}_{p}, \underline{\eta}\right)$. We will extensively make use of the identity (3.4). Let $v \in H^{1}\left(\Omega, \mathbb{R}^{3}\right)$ with $v_{\mid \Gamma_{D}}=0$. Multiply the equilibrium equation with $v-\dot{u}$ and integrate in space by parts and use the symmetry of $\sigma$ and the elasticity relation to get

$$
\int_{\Omega}\left\langle\mathbb{C}_{\mathrm{iso}} \operatorname{sym}(\nabla u-\bar{m} \underline{\gamma}), \operatorname{sym}(\nabla v-\nabla \dot{u})\right\rangle d x=\int_{\Omega} f(v-\dot{u}) d x .
$$

Now, for any $X \in C^{\infty}(\bar{\Omega}, \mathfrak{s l}(3))$ such that $X \times n=0$ on $\Gamma$ we integrate (3.12) over $\Omega$, integrate by parts the term with Curl Curl using the boundary conditions

$$
\left(X-\dot{\mathcal{X}}_{p}\right) \times n=0 \text { on } \Gamma_{\mathrm{D}}, \quad \operatorname{Curl} \mathcal{X}_{p} \times n=0 \text { on } \partial \Omega \backslash \Gamma_{\mathrm{D}}
$$


Well-posedness for the microcurl model

\begin{tabular}{|c|c|}
\hline Additive split of distortion: & $\nabla u=\underset{\substack{n_{\text {silp }} \\
n_{\text {sin }}}}{ }+p, \quad \varepsilon_{e}=\operatorname{sym} e, \quad \varepsilon_{p}=\operatorname{sym} p$ \\
\hline Plastic distortion in slip system: & $p=\sum_{\alpha=1} \gamma^{\alpha} m^{\alpha}$ with $m^{\alpha}=l^{\alpha} \otimes \nu^{\alpha}, \quad \operatorname{tr}(p)=0$ \\
\hline $\begin{array}{l}\text { Equilibrium: } \\
\text { Microbalance: }\end{array}$ & $\begin{array}{l}\text { Div } \sigma+f=0 \text { with } \sigma=\mathbb{C}_{\text {iso }} \varepsilon_{e}=\mathbb{C}_{\text {iso }}\left(\operatorname{sym} \nabla u-\varepsilon_{p}\right) \\
\mu L_{c}^{2} \text { Curl Curl } \mathcal{X}_{p}=-\mu H_{\chi}\left(\mathcal{X}_{p}-p\right)\end{array}$ \\
\hline Free energy: & $\begin{array}{l}\frac{1}{2}\left\langle\mathbb{C}_{\mathrm{iso}} \varepsilon^{e}, \varepsilon^{e}\right\rangle+\frac{1}{2} \mu H_{\chi}\left\|p-\mathcal{X}_{p}\right\|^{2} \\
\quad+\frac{1}{2} \mu L_{c}^{2}\left\|\operatorname{Curl} \mathcal{X}_{p}\right\|^{2}+\frac{1}{2} \mu k_{2} \sum_{\alpha}\left|\eta^{\alpha}\right|^{2}\end{array}$ \\
\hline $\begin{array}{l}\text { Yield condition in } \alpha \text {-th slip system: } \\
\text { where }\end{array}$ & $\begin{array}{l}\left|\tau_{\mathrm{E}}^{\alpha}\right|+g^{\alpha}-\sigma_{0} \leq 0 \\
\tau_{\mathrm{E}}^{\alpha}:=\left\langle\Sigma_{E}, m^{\alpha}\right\rangle \text { with } \\
\Sigma_{E}:=\sigma+\mu H_{\chi}\left(\mathcal{X}_{p}-p\right)=\sigma-\mu L_{c}^{2} \text { Curl Curl } \mathcal{X}_{p} \\
g^{\alpha}=-\mu k_{2} \eta^{\alpha}\end{array}$ \\
\hline Dissipation inequality in $\alpha$-th slip system: & $\tau_{\mathrm{E}}^{\alpha} \dot{\gamma}^{\alpha}+g^{\alpha} \dot{\eta}^{\alpha} \geq 0$ \\
\hline Dissipation function in $\alpha$-th slip system: & $\mathcal{D}_{\text {iso }}^{\alpha}\left(q^{\alpha}, \beta^{\alpha}\right):= \begin{cases}\sigma_{0}\left|q^{\alpha}\right| & \text { if }\left|q^{\alpha}\right| \leq \beta^{\alpha} \\
\infty & \text { otherwise }\end{cases}$ \\
\hline Flow rules in primal form: & $\left(\tau_{\mathrm{E}}^{\alpha}, g^{\alpha}\right) \in \partial \mathcal{D}_{\mathrm{iso}}^{\alpha}\left(\dot{\gamma}^{\alpha}, \dot{\eta}^{\alpha}\right)$ \\
\hline Flow rules in dual form: & $\dot{\gamma}^{\alpha}=\lambda^{\alpha} \frac{\tau_{\mathrm{E}}^{\alpha}}{\left|\tau_{\mathrm{E}}^{\alpha}\right|}$ \\
\hline $\begin{array}{l}\text { KKT conditions: } \\
\text { Boundary conditions for } \mathcal{X}_{p}: \\
\text { Function space for } \mathcal{X}_{p}:\end{array}$ & $\begin{array}{l}\lambda^{\alpha} \geq 0, \quad \phi\left(\tau_{E}^{\alpha}, g^{\alpha}\right) \leq 0, \quad \lambda^{\alpha} \phi\left(\tau_{\mathrm{E}}^{\alpha}, g^{\alpha}\right)=0 \\
\mathcal{X}_{p} \times n=0 \text { on } \Gamma_{\mathrm{D}}, \quad\left(\operatorname{Curl} \mathcal{X}_{p}\right) \times n=0 \text { on } \partial \Omega \backslash \Gamma_{\mathrm{D}} \\
\mathcal{X}_{p}(t, \cdot) \in \mathrm{H}\left(\operatorname{Curl} ; \Omega, \mathbb{R}^{3 \times 3}\right)\end{array}$ \\
\hline
\end{tabular}

Table 2: The microcurl model in single crystal gradient plasticity with isotropic hardening.

and get

$$
\int_{\Omega}\left[\mu L_{c}^{2}\left\langle\operatorname{Curl} \mathcal{X}_{p}, \operatorname{Curl} X-\operatorname{Curl} \dot{\mathcal{X}}_{p}\right\rangle+\mu H_{\chi}\left\langle\mathcal{X}_{p}-\bar{m} \underline{\gamma}, X-\dot{\mathcal{X}}_{p}\right\rangle\right] d x=0 .
$$

Moreover, for any $\underline{q}=\left(q^{1}, \ldots, q^{n_{\text {slip }}}\right)$ with $q^{\alpha} \in C^{\infty}(\bar{\Omega})$ and any $\underline{\beta}=\left(\beta^{1}, \ldots, \beta^{n_{\text {slip }}}\right)$ with $\beta^{\alpha} \in L^{2}(\Omega)$, summing (3.36) over $\alpha=1, \ldots, n_{\text {slip }}$ and integrating over $\Omega$, we get

$$
\begin{gathered}
\int_{\Omega} \mathcal{D}_{\text {iso }}(\underline{q}, \underline{\beta}) d x-\int_{\Omega} \mathcal{D}_{\text {iso }}(\underline{\dot{\gamma}}, \underline{\dot{\eta}}) d x-\int_{\Omega}\left\langle\mathbb{C}_{\text {iso }} \operatorname{sym}(\nabla u-\bar{m} \underline{\gamma}), \operatorname{sym}(\bar{m} \underline{q}-\bar{m} \underline{\dot{\gamma}})\right\rangle d x \\
+\int_{\Omega}\left[-\mu H_{\chi}\left\langle\mathcal{X}_{p}-\bar{m} \underline{\gamma}, \bar{m} \underline{q}-\bar{m} \underline{\dot{\gamma}}\right\rangle+\mu k_{2}\langle\underline{\eta}, \underline{\beta}-\underline{\dot{\eta}}\rangle\right] d x \geq 0 .
\end{gathered}
$$

where

$$
\mathcal{D}_{\text {iso }}(\underline{q}, \underline{\beta}):=\sum_{\alpha} \mathcal{D}_{\text {iso }}^{\alpha}\left(q^{\alpha}, \beta^{\alpha}\right) .
$$

Now adding up (4.23)-(4.25) we get the following weak formulation of the problem set in 
Section 3.2.4 in the form of a variational inequality:

$$
\begin{gathered}
\int_{\Omega}\left[\left\langle\mathbb{C}_{\text {iso }} \operatorname{sym}(\nabla u-\bar{m} \underline{\gamma}), \operatorname{sym}(\nabla v-\bar{m} \underline{q})-\operatorname{sym}(\nabla \dot{u}-\bar{m} \underline{\dot{\gamma}})\right\rangle+\mu L_{c}^{2}\left\langle\operatorname{Curl} \mathcal{X}_{p}, \operatorname{Curl} X-\operatorname{Curl} \dot{\mathcal{X}}_{p}\right\rangle\right. \\
\left.+\mu H_{\chi}\left\langle\mathcal{X}_{p}-\bar{m} \underline{\gamma},(X-\bar{m} \underline{q})-\left(\dot{\mathcal{X}}_{p}-\bar{m} \underline{\dot{\gamma}}\right)\right\rangle+\mu k_{2}\langle\underline{\eta}, \underline{\beta}-\underline{\dot{\eta}}\rangle\right] d x \\
\left.\quad+\int_{\Omega} \mathcal{D}_{\text {iso }} \underline{q}, \underline{\beta}\right) d x-\int_{\Omega} \mathcal{D}_{\text {iso }}(\underline{\dot{\gamma}}, \underline{\dot{\eta}}) d x \geq \int_{\Omega} f(v-\dot{u}) d x
\end{gathered}
$$

\subsubsection{Existence result for the weak formulation}

To prove the existence result for the weak formulation (3.41), we closely follow the abstract machinery developed by Han and Reddy in [48] for mathematical problems in geometrically linear classical plasticity and used for instance in [20, 86, 68, 24, 25] for models of gradient plasticity. To this aim, equation (3.41) is written as the variational inequality of the second kind: find $w=\left(u, \underline{\gamma}, \mathcal{X}_{p}, \underline{\eta}\right) \in \mathrm{H}^{1}(0, T ; \mathbf{Z})$ such that $w(0)=0, \dot{w}(t) \in \mathbf{W}$ for a.e. $t \in[0, T]$ and

$$
\boldsymbol{a}(w, z-\dot{w})+j(z)-j(\dot{w}) \geq\langle\ell, z-\dot{w}\rangle \text { for every } z \in \mathrm{W} \text { and for a.e. } t \in[0, T],
$$

where $\mathbf{Z}$ is a suitable Hilbert space and $\mathbf{W}$ is some closed, convex subset of $\mathbf{Z}$ to be constructed later,

$$
\begin{aligned}
\boldsymbol{a}(w, z)= & \int_{\Omega}\left[\left\langle\mathbb{C}_{\text {iso }} \operatorname{sym}(\nabla u-\bar{m} \underline{\gamma}), \operatorname{sym}(\nabla v-\bar{m} \underline{q})\right\rangle+\mu L_{c}^{2}\left\langle\operatorname{Curl} \mathcal{X}_{p}, \operatorname{Curl} X\right\rangle\right. \\
& \left.\quad+\mu H_{\chi}\left\langle\mathcal{X}_{p}-\bar{m} \underline{\underline{\gamma}}, X-\bar{m} \underline{q}\right\rangle+\mu k_{2}\langle\underline{\eta}, \underline{\beta}\rangle\right] d x, \\
j(z)= & \int_{\Omega} \mathcal{D}_{\text {iso }}(\underline{q}, \underline{\beta}) d x, \\
\langle\ell, z\rangle= & \int_{\Omega} f v d x,
\end{aligned}
$$

for $w=\left(u, \underline{\gamma}, \mathcal{X}_{p}, \underline{\eta}\right)$ and $z=(v, \underline{q}, X, \underline{\beta})$ in Z.

The Hilbert space $\mathbf{Z}$ and the closed convex subset $\mathbf{W}$ are constructed in such a way that the functionals $\boldsymbol{a}, j$ and $\ell$ satisfy the assumptions in the abstract result in [48, Theorem 6.19]. The key issue here is the coercivity of the bilinear form $\boldsymbol{a}$ on the set $\mathrm{W}$, that is, $a(z, z) \geq C\|z\|_{Z}^{2}$ for every $z \in \mathrm{W}$ and for some $C>0$.

We let

$$
\begin{aligned}
\mathrm{V} & :=\mathrm{H}_{0}^{1}\left(\Omega, \Gamma_{\mathrm{D}}, \mathbb{R}^{3}\right)=\left\{v \in \mathrm{H}^{1}\left(\Omega, \mathbb{R}^{3}\right) \mid v_{\mid \Gamma_{\mathrm{D}}}=0\right\} \\
\mathrm{P} & :=\mathrm{L}^{2}\left(\Omega, \mathbb{R}^{n_{\text {slip }}}\right) \\
\mathrm{Q} & :=\mathrm{H}_{0}\left(\mathrm{Curl} ; \Omega, \Gamma_{\mathrm{D}}, \mathfrak{s l}(3)\right) \\
\Lambda & :=L^{2}\left(\Omega, \mathbb{R}^{n_{\text {slip }}}\right) \\
\mathrm{Z} & :=\mathrm{V} \times \mathrm{P} \times \mathrm{Q} \times \Lambda \\
\mathrm{W} & :=\left\{z=(v, \underline{q}, X, \underline{\beta}) \in \mathrm{Z}|| q^{\alpha} \mid \leq \beta^{\alpha}, \quad \alpha=1, \ldots, n_{\text {slip }}\right\}
\end{aligned}
$$


and define the norms

$$
\begin{aligned}
& \|v\|_{V}:=\|\nabla v\|_{L^{2}}, \quad\|\underline{q}\|_{P}^{2}=\sum_{\alpha}\left\|q^{\alpha}\right\|_{L^{2}}^{2}, \quad\|\underline{\beta}\|_{\Lambda}^{2}=\sum_{\alpha}\left\|\beta^{\alpha}\right\|_{L^{2}}^{2}, \quad\|X\|_{Q}:=\|X\|_{\mathrm{H}(\mathrm{Curl} ; \Omega)}, \\
& \|z\|_{Z}^{2}:=\|v\|_{V}^{2}+\|\underline{q}\|_{P}^{2}+\|X\|_{Q}^{2}+\|\underline{\beta}\|_{\Lambda}^{2} \quad \text { for } z=(v, \underline{q}, X, \underline{\beta}) \in \mathbf{Z} .
\end{aligned}
$$

Let us show that the bilinear form $\boldsymbol{a}$ is coercive on $\mathrm{W}$. Let therefore $z=(v, \underline{q}, X, \underline{\beta}) \in \mathrm{W}$. First of all notice that

$$
\|\bar{m} \underline{q}\|_{L^{2}} \leq\|\underline{q}\|_{P} \leq\|\underline{\beta}\|_{\Lambda}
$$

So,

$$
\begin{aligned}
& \boldsymbol{a}(z, z) \geq m_{0}\|\operatorname{sym}(\nabla v-\bar{m} \underline{q})\|_{L^{2}}^{2}(\text { from }(2.3))+\mu H_{\chi}\|X-\bar{m} \underline{q}\|_{L^{2}}^{2}+\mu L_{c}^{2}\|\operatorname{Curl} X\|_{L^{2}}^{2} \\
& +\mu k_{2}\|\underline{\beta}\|_{\Lambda}^{2} \\
& =m_{0}\left[\|\operatorname{sym} \nabla v\|_{L^{2}}^{2}+\|\operatorname{sym}(\bar{m} \underline{q})\|_{2}^{2}-2\langle\operatorname{sym} \nabla v, \operatorname{sym}(\bar{m} \underline{q})\rangle_{L^{2}}\right]+\mu H_{\chi}\left[\|X\|_{L^{2}}^{2}\right. \\
& \left.+\|\bar{m} \underline{q}\|_{L^{2}}^{2}-2\langle X, \bar{m} \underline{q}\rangle_{L^{2}}\right]+\mu L_{c}^{2}\|\operatorname{Curl} X\|_{L^{2}}^{2}+\mu k_{2}\|\underline{\beta}\|_{\Lambda}^{2} \\
& \geq m_{0}\left[\|\operatorname{sym} \nabla v\|_{L^{2}}^{2}+\|\operatorname{sym}(\bar{m} \underline{q})\|_{L^{2}}^{2}-\theta\|\operatorname{sym}(\nabla v)\|_{L^{2}}^{2}-\frac{1}{\theta}\|\operatorname{sym}(\bar{m} \underline{q})\|_{L^{2}}^{2}\right] \\
& +\mu H_{\chi}\left[\|X\|_{L^{2}}^{2}+\|\bar{m} \underline{q}\|_{L^{2}}^{2}-\theta\|X\|_{L^{2}}^{2}-\frac{1}{\theta}\|\bar{m} \underline{q}\|_{L^{2}}^{2}\right] \text { (Young's inequality) } \\
& +\mu L_{c}^{2}\|\operatorname{Curl} X\|_{L^{2}}^{2}+\frac{1}{2} \mu k_{2}\|\underline{\beta}\|_{\Lambda}^{2}+\frac{1}{2} \mu k_{2}\|\underline{q}\|_{P}^{2} \quad \text { (using second } \leq \text { in }(3.53) \text { ) } \\
& =m_{0}(1-\theta)\|\operatorname{sym} \nabla v\|_{L^{2}}^{2}+m_{0}\left(1-\frac{1}{\theta}\right)\|\operatorname{sym}(\bar{m} \underline{q})\|_{L^{2}}^{2}+\mu H_{\chi}\left(1-\frac{1}{\theta}\right)\|\underline{q}\|_{P}^{2} \\
& +\frac{1}{2} \mu k_{2}\|\underline{q}\|_{P}^{2}+\mu H_{\chi}(1-\theta)\|X\|_{L^{2}}^{2}+\mu L_{c}^{2}\|\operatorname{Curl} X\|_{2}^{2}+\frac{1}{2} \mu k_{2}\|\underline{\beta}\|_{\Lambda}^{2} \\
& \geq m_{0}(1-\theta)\|\operatorname{sym} \nabla v\|_{L^{2}}^{2}+\left[\left(m_{0}+\mu H_{\chi}\right)\left(1-\frac{1}{\theta}\right)+\frac{1}{2} \mu k_{2}\right]\|\underline{q}\|_{P}^{2} \\
& +\mu H_{\chi}(1-\theta)\|X\|_{L^{2}}^{2}+\mu L_{c}^{2}\|\operatorname{Curl} X\|_{2}^{2}+\frac{1}{2} \mu k_{2}\|\underline{\beta}\|_{\Lambda}^{2} \quad(\text { for } 0<\theta<1) \text {. }
\end{aligned}
$$

So, since the hardening constant $k_{2}>0$, it is possible to choose $\theta$ such that

$$
\frac{m_{0}+\mu H_{\chi}}{m_{0}+\mu H_{\chi}+\frac{1}{2} \mu k_{2}}<\theta<1
$$

we always are able to find some constant $C\left(\theta, m_{0}, \mu, H_{\chi}, k_{2}, L_{c}, \Omega\right)>0$ such that

$$
a(z, z) \geq C\left[\|v\|_{V}^{2}+\|\underline{q}\|_{P}^{2}+\|X\|_{\mathrm{H}(\mathrm{Curl} ; \Omega)}^{2}+\|\underline{\beta}\|_{\Lambda}^{2}\right]=C\|z\|_{Z}^{2} \quad \forall z=(v, \underline{q}, X, \underline{\beta}) \in \mathrm{W} .
$$

This shows existence for the microcurl model in single gradient plasticity with isotropic hardening. 


\subsubsection{Uniqueness of the weak/strong solution}

As shown in [22] for a canonical rate-independent model of geometrically linear isotropic gradient plasticity with isotropic hardening and plastic spin, the uniqueness of the solution for our model can be obtained similarly. To this aim, notice that if $\left(u, \underline{\gamma}, \mathcal{X}_{p}, \underline{\eta}\right)$ is a weak solution of the model, then $\left(u, \underline{\gamma}, \mathcal{X}_{p}, \underline{\eta}\right)$ is also a strong solution. In fact, choosing appropriatetly test functions in the variational inequality (3.41), we obtain both equilibrium and microbalance equations on the one hand. The latter which is

$$
\mu L_{c}^{2} \operatorname{Curl} \operatorname{Curl} \mathcal{X}_{p}=-\mu H_{\chi}\left(\mathcal{X}_{p}-p\right)
$$

is satisfied first in the distributional sense and hence is satisfied also in the $L^{2}$-sense since the right hand side is in $L^{2}\left(\Omega, \mathbb{R}^{3 \times 3}\right)$. Therefore, it follows that Curl Curl $\mathcal{X}_{p}$ is also in $L^{2}\left(\Omega, \mathbb{R}^{3 \times 3}\right)$. Now going back to (3.41), we also derive the boundary condition $\left(\operatorname{Curl} \mathcal{X}_{p}\right) \times\left. n\right|_{\partial \Omega \backslash \Gamma_{\mathrm{D}}}=0$ which is now justified because we derived that $\operatorname{Curl} \mathcal{X}_{p} \in \mathrm{H}(\operatorname{Curl} ; \Omega$, sl( $(3))$.

On the other hand, we also obtain from (3.41) the following set of inequatlities

$$
\left\langle\dot{\Gamma}_{p}^{\alpha}, \Sigma^{\alpha}-\Sigma_{p}^{\alpha}\right\rangle \leq 0 \quad \forall \Sigma^{\alpha} \in \mathcal{K}^{\alpha}, \quad \alpha=1, \ldots, n_{\text {silp }}
$$

and hence, $\left(u, \underline{\gamma}, \mathcal{X}_{p}, \underline{\eta}\right)$ is a strong solution.

Now let us consider two solutions $w_{i}:=\left(u_{i}, \underline{\gamma_{i}}, \mathcal{X}_{p i}, \underline{\eta_{i}}\right), i=1,2$ of (3.41) satisfying the same initial conditions, let $\Gamma_{p_{i}}^{\alpha}=\left(\gamma_{i}^{\alpha}, \eta_{i}^{\alpha}\right)$ and $\Sigma_{p i}^{\alpha}:=\left(\tau_{E_{i}}^{\alpha}, \overline{g_{i}^{\alpha}}\right)$ be the corresponding stresses. That is,

$$
\begin{aligned}
\tau_{E_{i}}^{\alpha} & =\left\langle\Sigma_{E_{i}}, m^{\alpha}\right\rangle=\left\langle\sigma_{i}+\mu H_{\chi}\left(\mathcal{X}_{p_{i}}-p_{i}\right), m^{\alpha}\right\rangle=\left\langle\sigma_{i}-\mu H_{\chi} \operatorname{Curl} \text { Curl } \mathcal{X}_{p_{i}}, m^{\alpha}\right\rangle, \\
g_{i}^{\alpha} & =-\mu k_{2} \eta_{i}^{\alpha}
\end{aligned}
$$

so that $\Gamma_{p_{i}}^{\alpha}$ and $\Sigma_{p_{i}}^{\alpha}$ satisfy

$$
\left\langle\dot{\Gamma}_{p_{1}}^{\alpha}, \Sigma^{\alpha}-\Sigma_{p_{1}}^{\alpha}\right\rangle \leq 0 \quad \text { and } \quad\left\langle\dot{\Gamma}_{p_{2}}^{\alpha}, \Sigma^{\alpha}-\Sigma_{p_{2}}^{\alpha}\right\rangle \leq 0 \quad \forall \Sigma^{\alpha} \in \mathcal{K}^{\alpha} .
$$

Now choose $\Sigma^{\alpha}=\Sigma_{p_{2}}^{\alpha}$ in $(3.59)_{1}$ and $\Sigma^{\alpha}=\Sigma_{p_{1}}^{\alpha}$ in (3.59) $)_{2}$ and add up to get

$$
\left\langle\Sigma_{p_{2}}^{\alpha}-\Sigma_{p_{1}}^{\alpha}, \dot{\Gamma}_{p_{1}}^{\alpha}-\dot{\Gamma}_{p_{2}}^{\alpha}\right\rangle \leq 0
$$

That is,

$$
\begin{gathered}
\left\langle\sigma_{2}-\sigma_{1}, m^{\alpha} \dot{\gamma}_{1}^{\alpha}-m^{\alpha} \dot{\gamma}_{2}^{\alpha}\right\rangle+\mu H_{\chi}\left\langle\left(\mathcal{X}_{p_{2}}-\mathcal{X}_{p_{1}}\right)-\left(p_{2}-p_{1}\right), m^{\alpha} \dot{\gamma}_{1}^{\alpha}-m^{\alpha} \dot{\gamma}_{2}^{\alpha}\right\rangle \\
+\left(g_{2}^{\alpha}-g_{1}^{\alpha}\right)\left(\dot{\eta}_{1}^{\alpha}-\dot{\eta}_{2}^{\alpha}\right) \leq 0
\end{gathered}
$$

and adding up over $\alpha$, we get

$$
\left\langle\sigma_{2}-\sigma_{1}, \dot{p}_{1}-\dot{p}_{2}\right\rangle+\mu H_{\chi}\left\langle\left(\mathcal{X}_{p_{2}}-\mathcal{X}_{p_{1}}\right)-\left(p_{2}-p_{1}\right), \dot{p}_{1}-\dot{p}_{2}\right\rangle+\left\langle\underline{g_{2}}-\underline{g_{1}}, \underline{\dot{\eta}_{1}}-\underline{\dot{\eta}_{2}}\right\rangle \leq 0
$$

Now, substitute $\operatorname{sym} p_{i}=\operatorname{sym} \nabla u_{i}-\mathbb{C}_{\text {iso }}^{-1} \sigma_{i}$ obtained from the elasticity relation, into the ex-

pression $\left\langle\sigma_{2}-\sigma_{1}, \dot{p}_{1}-\dot{p}_{2}\right\rangle$ and $\dot{p}_{i}=\dot{\mathcal{X}}_{p i}+\frac{L_{c}^{2}}{H_{\chi}}$ Curl Curl $\dot{\mathcal{X}}_{p_{i}}$ obtained from the microbalance equation into the expression $\left\langle\mathcal{X}_{p_{2}}-\mathcal{X}_{p_{1}}, \dot{p}_{1}-\dot{p}_{2}\right\rangle$ and get from (3.62) that 


$$
\begin{gathered}
\left\langle\sigma_{2}-\sigma_{1}, \mathbb{C}_{\text {iso }}^{-1}\left(\dot{\sigma}_{2}-\dot{\sigma}_{1}\right)\right\rangle+\mu H_{\chi}\left\langle p_{1}-p_{2}, \dot{p}_{1}-\dot{p}_{2}\right\rangle+\mu H_{\chi}\left\langle\mathcal{X}_{p_{2}}-\mathcal{X}_{p_{1}}, \dot{\mathcal{X}}_{p_{1}}-\dot{\mathcal{X}}_{p_{2}}\right\rangle \\
+\mu L_{c}^{2}\left\langle\mathcal{X}_{p_{2}}-\mathcal{X}_{p_{1}}, \operatorname{Curl} \operatorname{Curl} \mathcal{X}_{p_{1}}-\operatorname{Curl} \operatorname{Curl} \mathcal{X}_{p_{2}}\right\rangle+\left\langle\underline{g_{2}}-\underline{g_{1}}, \underline{\dot{\eta}_{1}}-\underline{\dot{\eta}_{2}}\right\rangle \\
\leq\left\langle\sigma_{1}-\sigma_{2}, \operatorname{sym}\left(\nabla u_{1}\right)-\operatorname{sym}\left(\nabla u_{2}\right)\right\rangle
\end{gathered}
$$

Now for every $t \in[0, T]$, we integrate (3.63) over $\Omega \times(0, t)$ using the boundary conditions on $\mathcal{X}_{p_{i}}$ and using the fact that

$$
\int_{\Omega}\left\langle\sigma_{1}-\sigma_{2}, \operatorname{sym} \nabla u_{1}-\operatorname{sym} \nabla u_{2}\right\rangle d x=0
$$

we get

$$
\begin{gathered}
\int_{0}^{t} \frac{d}{d s}\left[\left\|\mathbb{C}_{\text {iso }}^{-1 / 2}\left(\sigma_{2}(s)-\sigma_{1}(s)\right)\right\|_{L^{2}}^{2}+\mu H_{\chi}\left\|p_{1}(s)-p_{2}(s)\right\|_{L^{2}}^{2}-\mu H_{\chi}\left\|\mathcal{X}_{p_{2}}(s)-\mathcal{X}_{p_{1}}(s)\right\|_{L^{2}}^{2}\right. \\
\left.-\mu L_{c}^{2}\left\|\operatorname{Curl} \mathcal{X}_{p_{2}}(s)-\operatorname{Curl} \mathcal{X}_{p_{1}}(s)\right\|_{L^{2}}^{2}+\mu k_{2}\left\|\underline{\eta_{1}}(s)-\underline{\eta_{2}}(s)\right\|_{L^{2}}^{2}\right] d s \leq 0 .
\end{gathered}
$$

Therefore, we obtain

$$
\begin{aligned}
\left\|\mathbb{C}_{\text {iso }}^{-1 / 2}\left(\sigma_{2}-\sigma_{1}\right)\right\|_{L^{2}}^{2}+\mu H_{\chi}\left\|p_{1}-p_{2}\right\|_{L^{2}}^{2}+\mu k_{2}\left\|\underline{\eta_{1}}-\underline{\eta_{2}}\right\|_{L^{2}}^{2} \\
\quad \leq \mu H_{\chi}\left\|\mathcal{X}_{p_{2}}-\mathcal{X}_{p_{1}}\right\|_{L^{2}}^{2}+\mu L_{c}^{2}\left\|\operatorname{Curl} \mathcal{X}_{p_{2}}-\operatorname{Curl} \mathcal{X}_{p_{1}}\right\|_{L^{2}}^{2} .
\end{aligned}
$$

On the other hand, we write the micro-balance equation for $p_{i}$ and $\mathcal{X}_{p_{i}}$ with $i=1,2$, as

$$
\begin{aligned}
& \mu H_{\chi} p_{1}=\mu L_{c}^{2} \text { Curl Curl } \mathcal{X}_{p_{1}}+\mu H_{\chi} \mathcal{X}_{p_{1}}, \\
& \mu H_{\chi} p_{2}=\mu L_{c}^{2} \text { Curl Curl } \mathcal{X}_{p_{2}}+\mu H_{\chi} \mathcal{X}_{p_{2}},
\end{aligned}
$$

then we subtract, take the scalar product with $\mathcal{X}_{p_{1}}-\mathcal{X}_{p_{2}}$, integrate using the boundary condition

$$
\left(\mathcal{X}_{p_{1}}-\mathcal{X}_{p_{2}}\right) \times\left. n\right|_{\Gamma_{\mathrm{D}}}=0 \quad \text { and } \quad\left(\operatorname{Curl} \mathcal{X}_{p_{1}}-\operatorname{Curl} \mathcal{X}_{p_{2}}\right) \times\left. n\right|_{\partial \Omega \backslash \Gamma_{\mathrm{D}}}=0
$$

and get

$$
\mu H_{\chi} \int_{\Omega}\left\langle p_{1}-p_{2}, \mathcal{X}_{p_{1}}-\mathcal{X}_{p_{2}}\right\rangle d x=\mu L_{c}^{2}\left\|\operatorname{Curl} \mathcal{X}_{p_{1}}-\operatorname{Curl} \mathcal{X}_{p_{2}}\right\|_{L^{2}}^{2}+\mu H_{\chi}\left\|\mathcal{X}_{p_{1}}-\mathcal{X}_{p_{2}}\right\|_{L^{2}}^{2}
$$

Therefore, we obtain from (3.64) and (3.67) that

$$
\begin{aligned}
\mu H_{\chi}\left\|p_{1}-p_{2}\right\|_{L^{2}}^{2} & \leq \mu L_{c}^{2}\left\|\operatorname{Curl} \mathcal{X}_{p_{1}}-\operatorname{Curl} \mathcal{X}_{p_{2}}\right\|_{L^{2}}^{2}+\mu H_{\chi}\left\|\mathcal{X}_{p_{1}}-\mathcal{X}_{p_{2}}\right\|_{L^{2}}^{2} \\
& \leq \mu H_{\chi}\left\|p_{1}-p_{2}\right\|_{L^{2}}\left\|\mathcal{X}_{p_{1}}-\mathcal{X}_{p_{2}}\right\|_{L^{2}}
\end{aligned}
$$

which implies that

$$
\left\|p_{1}-p_{2}\right\|_{L^{2}}=\left\|\mathcal{X}_{p_{1}}-\mathcal{X}_{p_{2}}\right\|_{L^{2}} \quad \text { and hence, } \quad\left\|\operatorname{Curl} \mathcal{X}_{p_{1}}-\operatorname{Curl} \mathcal{X}_{p_{2}}\right\|_{L^{2}}=0
$$


Now, going back to (3.64), we get

$$
\left\|\mathbb{C}_{\text {iso }}^{-1 / 2}\left(\sigma_{2}-\sigma_{1}\right)\right\|_{L^{2}}^{2}+\mu k_{2}\left\|\underline{\eta_{1}}-\underline{\eta_{2}}\right\|_{L^{2}}^{2} \leq 0 .
$$

Hence, we obtain so far,

$$
\sigma_{1}-\sigma_{2}, \quad \underline{\eta_{1}}=\underline{\eta_{2}} \quad \text { and } \quad \operatorname{Curl} \mathcal{X}_{p_{1}}=\operatorname{Curl} \mathcal{X}_{p_{2}} \quad \Rightarrow \quad \tau_{E_{1}}^{\alpha}=\tau_{E_{2}}^{\alpha} .
$$

Now, let us prove that $\gamma_{1}^{\alpha}=\gamma_{2}^{\alpha}$ for every $\alpha$. In fact, from the definition of the normal cone it follows that $\dot{\Gamma}_{p_{i}}^{\alpha}=0$ that is, $\dot{\gamma}_{i}^{\alpha}=\dot{\eta}_{i}^{\alpha}=0$ inside the elastic domain $\operatorname{Int}\left(\mathcal{K}^{\alpha}\right)$ (for the $\alpha$-slip system), which from the initial conditions imply that $\gamma_{i}^{\alpha}=0$ inside $\operatorname{Int}\left(\mathcal{K}^{\alpha}\right)$. Now, looking at the flow rule in dual form (for the $\alpha$-slip system) in Table 2, we obtain from $\tau_{E_{1}}^{\alpha}=\tau_{E_{2}}^{\alpha}$ that $\dot{\gamma}_{1}^{\alpha}=\dot{\gamma}_{2}^{\alpha}$ which implies that $\gamma_{1}^{\alpha}=\gamma_{2}^{\alpha}$ from the initial conditions. Therefore, we obtain $p_{1}=p_{2}$ which implies from $(3.70)_{1}$ that $\mathcal{X}_{p_{1}}=\mathcal{X}_{p_{2}}$.

Now, it remains to show that $u_{1}=u_{2}$. This is obtained exactly as in [22]. We repeat the proof here just for the reader's convenience. To this end, we use $\operatorname{sym}\left(\nabla u_{i}\right)=\mathbb{C}^{-1} \sigma_{i}+\operatorname{sym} p_{i}$ obtained from the elasticity relation and get

$$
\operatorname{sym}\left(\nabla\left(u_{1}-u_{2}\right)\right)=\mathbb{C}^{-1}\left(\sigma_{1}-\sigma_{2}\right)+\operatorname{sym}\left(p_{1}-p_{2}\right)=0,
$$

and hence, from the first Korn's inequality (see e.g. [62]), we get $\nabla\left(u_{1}-u_{2}\right)=0$ which implies that $u_{1}=u_{2}$. Therefore, we finally obtain

$$
u_{1}=u_{2}, \quad \sigma_{1}=\sigma_{2}, \quad\left(\underline{\gamma_{1}}=\underline{\gamma_{2}} \Rightarrow p_{1}=p_{2}\right), \quad \mathcal{X}_{p_{1}}=\mathcal{X}_{p_{2}}, \quad \underline{\eta_{1}}=\underline{\eta_{2}},
$$

and thus the uniqueness of a weak/strong solution.

Remark 3.1 It should be stressed that, in the proof, $k_{2}>0$ is necessary for uniqueness of the displacement field (and of the slip variables). If $k_{2}=0$, we have perfect plasticity and multiple solutions involving displacement discontinuities along slip lines, as in conventional Hill's plasticity, are possible. The curl operator does not regularize such discontinuities since curl $p$ may vanish in the presence of gradient of slip $\gamma^{\alpha}$ perpendicularly to the slip planes. It is shown in [30] that gradient models lead to finite width kink bands but still allow for slip band discontinuities, parallel to slip planes, in single crystals.

\subsection{The model with linear kinematical hardening}

Here we consider the model where the isotropic hardening has been replaced with linear kinematical hardening.

\subsubsection{The description of the model}

Here the free-energy density $\Psi$ is also given in the additively separated form as

$$
\begin{aligned}
& \Psi\left(\nabla u, p, \mathcal{X}_{p}, \operatorname{Curl} \mathcal{X}_{p}\right):=\underbrace{\Psi_{e}^{\operatorname{lin}}\left(\varepsilon_{e}\right)}_{\text {elastic energy }}+\underbrace{\Psi_{\text {micro }}^{\operatorname{lin}}\left(p, \mathcal{X}_{p}\right)}_{\text {micro energy }} \\
& +\underbrace{\Psi_{\text {curl }}^{\operatorname{lin}\left(\operatorname{Curl} \mathcal{X}_{p}\right)}}_{\text {defect-like energy }(\mathrm{GND})}+\underbrace{\Psi_{\text {kin }}^{\operatorname{lin}\left(\varepsilon_{p}\right)}}_{\text {hardening energy }(\mathrm{SSD})},
\end{aligned}
$$


where

$$
\begin{aligned}
\Psi_{e}^{\operatorname{lin}}\left(\varepsilon_{e}\right) & :=\frac{1}{2}\left\langle\varepsilon_{e}, \mathbb{C}_{\mathrm{iso}} \varepsilon_{e}\right\rangle=\frac{1}{2}\left\langle\operatorname{sym}(\nabla u-p), \mathbb{C}_{\text {iso }} \operatorname{sym}(\nabla u-p)\right\rangle, \\
\Psi_{\text {micro }}^{\operatorname{lin}}\left(p, \mathcal{X}_{p}\right) & :=\frac{1}{2} \mu H_{\chi}\left\|p-\mathcal{X}_{p}\right\|^{2}, \quad \Psi_{\text {curl }}^{\operatorname{lin}}\left(\operatorname{Curl} \mathcal{X}_{p}\right):=\frac{1}{2} \mu L_{c}^{2}\left\|\operatorname{Curl} \mathcal{X}_{p}\right\|^{2}, \\
\Psi_{\text {kin }}^{\operatorname{lin}}\left(\varepsilon_{p}\right) & :=\frac{1}{2} \mu k_{1}\left\|\varepsilon_{p}\right\|^{2}=\frac{1}{2} \mu k_{1}\|\operatorname{sym} p\|^{2} .
\end{aligned}
$$

In this case, the equilibrium equation and the microcurl balance are obtained as in (3.10) and in (3.12) respectively.

Now, the free-energy imbalance

$$
\dot{\Psi} \leq\langle\sigma, \nabla u\rangle=\left\langle\sigma, \dot{\varepsilon}_{e}\right\rangle+\langle\sigma, \dot{p}\rangle
$$

and the expansion of $\dot{\Psi}$ lead to the usual infinitesimal eleastic stress-strain relation

$$
\sigma=2 \mu \operatorname{sym}(\nabla u-p)+\lambda \operatorname{tr}(\nabla u-p) \mathbb{1}=2 \mu\left(\operatorname{sym}(\nabla u)-\varepsilon_{p}\right)+\lambda \operatorname{tr}(\nabla u) \mathbb{1}
$$

and the local reduced dissipation inequality

$$
\left\langle\Sigma_{E}, \dot{p}\right\rangle \geq 0,
$$

where the non-symmetric Eshelby-type stress tensor in this case takes the form

$$
\Sigma_{E}:=\sigma+\Sigma_{\text {micro }}^{\operatorname{lin}}+\Sigma_{\text {kin }}^{\operatorname{lin}}
$$

with

$$
\begin{aligned}
\sum_{\text {micro }}^{\operatorname{lin}} & :=\mu H_{\chi}\left(\mathcal{X}_{p}-p\right)=-\mu L_{c}^{2} \operatorname{Curl} \operatorname{Curl} \mathcal{X}_{p}, \\
\sum_{\text {kin }}^{\operatorname{lin}} & :=-\mu k_{1} \varepsilon_{p}=-\mu k_{1} \operatorname{sym} p .
\end{aligned}
$$

Two sources of kinematic hardening therefore arise in the model: the size-dependent contribution, $\Sigma_{\text {micro }}^{\operatorname{lin}}$, induced by strain gradient plasticity, and conventional size-independent linear kinematic hardening $\sum_{\text {kin }}^{\operatorname{lin}}$.

Following the steps in the derivation of the strong formulation of the microcurl model with isotropic hardening in Section 3.2.4, we get the strong formulation in Table 3 for the model with linear kinematical hardening.

\subsubsection{The weak formulation of the model with linear kinematical hardening}

The equilibrium and microbalance equations in weak form are

$$
\begin{aligned}
& \int_{\Omega}\left\langle\mathbb{C}_{\text {iso }}\left(\operatorname{sym} \nabla u-\varepsilon_{p}\right), \operatorname{sym}(\nabla v-\nabla \dot{u})\right\rangle d x=\int_{\Omega} f(v-\dot{u}) d x, \\
& \int_{\Omega}\left[\mu L_{c}^{2}\left\langle\operatorname{Curl} \mathcal{X}_{p}, \operatorname{Curl} X-\operatorname{Curl} \dot{\mathcal{X}}_{p}\right\rangle+\mu H_{\chi}\left\langle\mathcal{X}_{p}-p, X-\dot{\mathcal{X}}_{p}\right\rangle\right] d x=0,
\end{aligned}
$$

for every $v \in \mathrm{V}$ and $X \in \mathrm{Q}$ with $\mathrm{V}$ and $\mathrm{Q}$ defined in (4.31) and in (4.33), respectively. 


\begin{tabular}{|c|c|}
\hline $\begin{array}{l}\text { Additive split of distortion: } \\
\text { Equilibrium: } \\
\text { Microbalance: }\end{array}$ & $\begin{array}{l}\nabla u=e+p, \quad \varepsilon_{e}=\operatorname{sym} e, \quad \varepsilon_{p}=\operatorname{sym} p \\
\operatorname{Div} \sigma+f=0 \text { with } \sigma=\mathbb{C}_{\text {iso }} \varepsilon^{e}=\mathbb{C}_{\text {iso }}\left(\operatorname{sym} \nabla u-\varepsilon_{p}\right) \\
\mu L_{c}^{2} \text { Curl Curl } \mathcal{X}_{p}=-\mu H_{\chi}\left(\mathcal{X}_{p}-p\right),\end{array}$ \\
\hline Free energy: & $\begin{array}{l}\frac{1}{2}\left\langle\mathbb{C}_{\mathrm{iso}} \varepsilon_{e}, \varepsilon_{e}\right\rangle+\frac{1}{2} \mu H_{\chi}\left\|p-\mathcal{X}_{p}\right\|^{2} \\
\quad+\frac{1}{2} \mu L_{c}^{2}\left\|\operatorname{Curl} \mathcal{X}_{p}\right\|^{2}+\frac{1}{2} \mu k_{1}\|\operatorname{sym} p\|^{2}\end{array}$ \\
\hline $\begin{array}{l}\text { Yield condition: } \\
\text { where }\end{array}$ & $\begin{array}{l}\phi\left(\Sigma_{E}\right):=\left\|\operatorname{dev} \Sigma_{E}\right\|-\sigma_{0} \leq 0 \\
\Sigma_{E}:=\sigma+\mu H_{\chi}\left(\mathcal{X}_{p}-p\right)-\mu k_{1} \operatorname{sym} p\end{array}$ \\
\hline $\begin{array}{l}\text { Dissipation inequality: } \\
\text { Dissipation function: } \\
\text { Flow rule in primal form: }\end{array}$ & $\begin{array}{l}\left\langle\Sigma_{E}, \dot{p}\right\rangle \geq 0 \\
\mathcal{D}_{\text {iso }}(q):=\sigma_{0}\|q\| \\
\Sigma_{E} \in \partial \mathcal{D}_{\text {iso }}(\dot{p})\end{array}$ \\
\hline Flow rule in dual form: & $\dot{p}=\lambda \frac{\operatorname{dev} \Sigma_{E}}{\left\|\operatorname{dev} \Sigma_{E}\right\|}$ \\
\hline $\begin{array}{l}\text { KKT conditions: } \\
\text { Boundary conditions for } \mathcal{X}_{p}: \\
\text { Function space for } \mathcal{X}_{p}:\end{array}$ & $\begin{array}{l}\lambda \geq 0, \quad \phi\left(\Sigma_{E}, g\right) \leq 0, \quad \lambda \phi\left(\Sigma_{E}, g\right)=0 \\
\mathcal{X}_{p} \times n=0 \text { on } \Gamma_{\mathrm{D}}, \quad\left(\operatorname{Curl} \mathcal{X}_{p}\right) \times n=0 \text { on } \partial \Omega \backslash \Gamma_{\mathrm{D}} \\
\mathcal{X}_{p}(t, \cdot) \in \mathrm{H}\left(\mathrm{Curl} ; \Omega, \mathbb{R}^{3 \times 3}\right)\end{array}$ \\
\hline
\end{tabular}

Table 3: The microcurl model in single crystal gradient plasticity with linear kinematical hardening.

Now, the primal formulation of the flow rule $\left(\Sigma_{E} \in \partial \mathcal{D}_{\text {kin }}(\dot{p})\right)$ in weak form reads for every $q \in L^{2}(\Omega, \mathfrak{s l}(3))$ as

$$
\begin{aligned}
\int_{\Omega} \mathcal{D}_{\text {kin }}(q) d x-\int_{\Omega} \mathcal{D}_{\text {kin }}(\dot{p}) d x \geq & \int_{\Omega}\left\langle\Sigma_{E}, q-\dot{p}\right\rangle d x \\
= & \int_{\Omega}\left\langle\mathbb{C}_{\text {iso }} \operatorname{sym}(\nabla u-p), \operatorname{sym}(q-\dot{p}\rangle d x\right. \\
& +\int_{\Omega}\left[\left\langle\mu H_{\chi}\left(\mathcal{X}_{p}-p\right)-\mu k_{1} \operatorname{sym} p, q-\dot{p}\right\rangle\right] d x .
\end{aligned}
$$

Now adding up (3.78), (3.79) and (3.80) we get the following weak formulation of the microcurl model of single crystal strain gradient plasticity with linear kinematical hardening in the form of a variational inequality:

$$
\begin{aligned}
\int_{\Omega}\left[\left\langle\mathbb{C}_{\text {iso }}\right.\right. & \operatorname{Sym}(\nabla u-p), \operatorname{sym}(\nabla v-q)-\operatorname{sym}(\nabla \dot{u}-\dot{p})\rangle+\mu L_{c}^{2}\left\langle\operatorname{Curl} \mathcal{X}_{p}, \operatorname{Curl} X-\operatorname{Curl} \dot{\mathcal{X}}_{p}\right\rangle \\
& \left.+\mu H_{\chi}\left\langle\mathcal{X}_{p}-p,(X-q)-\left(\dot{\mathcal{X}}_{p}-\dot{p}\right)\right\rangle+\mu k_{1}\langle\operatorname{sym} p, \operatorname{sym}(q-\dot{p})\rangle\right] d x \\
& +\int_{\Omega} \mathcal{D}_{\text {kin }}(q) d x-\int_{\Omega} \mathcal{D}_{\text {kin }}(\dot{p}) d x \geq \int_{\Omega} f(v-\dot{u}) d x
\end{aligned}
$$

That is, setting $\mathrm{Z}:=\mathrm{V} \times \mathrm{P} \times \mathrm{Q}$ with $\mathrm{V}, \mathrm{P}$ and $\mathrm{Q}$ defined in (4.31)-(4.33) and their norms in (4.37), we get the problem of the form: Find $w=\left(u, p, \mathcal{X}_{p}\right) \in \mathrm{H}^{1}(0, T ; Z)$ such that $w(0)=0$ and

$$
\boldsymbol{a}(\dot{w}, z-w)+j(z)-j(\dot{w}) \geq\langle\ell, z-\dot{w}\rangle \text { for every } z \in \mathbf{Z} \text { and for a.e. } t \in[0, T],
$$


where

$$
\begin{aligned}
& \boldsymbol{a}(w, z):=\int_{\Omega}\left[\left\langle\mathbb{C}_{\text {iso }} \operatorname{sym}(\nabla u-p), \operatorname{sym}(\nabla v-q)\right\rangle+\mu L_{c}^{2}\left\langle\operatorname{Curl} \mathcal{X}_{p}, \operatorname{Curl} X\right\rangle\right. \\
&\left.+\mu H_{\chi}\left\langle\mathcal{X}_{p}-p, X-q\right\rangle+\mu k_{1}\langle\operatorname{sym} p, \operatorname{sym} q\rangle\right] d x, \\
& j(z):=\int_{\Omega} \mathcal{D}_{\text {kin }}(q) d x, \\
&\langle\ell, z\rangle:=\int_{\Omega} f v d x,
\end{aligned}
$$

for $w=\left(u, p, \mathcal{X}_{p}\right)$ and $z=(v, q, \mathrm{Q})$ in $\mathrm{Z}$.

\subsubsection{Existence and uniqueness for the model with linear kinematic hardening}

In order to show the existence and uniqueness for the problem in (3.82)-(3.85) using [48, Theorem 6.15], we only need to show here that the bilinear form $\boldsymbol{a}$ is Z-coercive. However, this is obtained following a different approach. We will make use of the following result.

Lemma 3.1 The mapping $\|\cdot\|_{*}: \mathrm{P} \times \mathrm{Q} \rightarrow[0, \infty)$ defined by

$$
\|(q, X)\|_{*}^{2}:=\|q-X\|_{L^{2}}^{2}+\|\operatorname{sym} q\|_{L^{2}}^{2}+\|\operatorname{Curl} X\|_{L^{2}}^{2}
$$

is a norm on $\mathrm{P} \times \mathrm{Q}$ equivalent to the norm defined by

$$
\|(q, X)\|_{P \times Q}^{2}=\|q\|_{L^{2}}^{2}+\|X\|_{H(\operatorname{Curl} ; \Omega)}^{2} .
$$

Proof. To show that $\|\cdot\|_{*}$ is a norm on $\mathrm{P} \times \mathrm{Q}$, we only check the vanishing property of a norm since the other properties are trivially satisfied. The vanishing property is obtained through the Korn-type inequality for incompatible tensor fields established in [72, 73, 74, 75], namely

$$
\|X\|_{L^{2}}^{2} \leq C\left(\|\operatorname{sym} X\|_{L^{2}}^{2}+\|\operatorname{Curl} X\|_{L^{2}}^{2}\right) \quad \forall X \in \mathrm{Q}:=\mathrm{H}_{0}\left(\operatorname{Curl} ; \Omega, \Gamma_{\mathrm{D}}, \mathbb{R}^{3 \times 3}\right) .
$$

In fact, let $(q, X) \in \mathrm{P} \times \mathrm{Q}$ be such that $\|(q, X)\|_{*}=0$, that is, $q=X$, sym $q=0$ and Curl $X=0$. Thus we get $\operatorname{sym} X=0$ and $\operatorname{Curl} X=0$. From (3.88), we then get $X=0$ and hence also $q=0$.

Now to show that both norms are equivalent, we will first show that $\left(\mathrm{P} \times \mathrm{Q},\|\cdot\|_{*}\right)$ is a Banach space. To this aim, let $\left(q_{n}, X_{n}\right)$ be a Cauchy sequence in $\left(\mathrm{P} \times \mathrm{Q},\|\cdot\|_{*}\right)$. Hence, the sequences $\left(q_{n}-X_{n}\right),\left(\operatorname{sym} q_{n}\right)$ and $\left(\operatorname{Curl} X_{n}\right)$ are all Cauchy in $L^{2}\left(\Omega, \mathbb{R}^{3 \times 3}\right)$ and therefore, there exist $A, B, C \in L^{2}\left(\Omega, \mathbb{R}^{3 \times 3}\right)$ such that

$$
q_{n}-X_{n} \rightarrow A, \quad \operatorname{sym} q_{n} \rightarrow B, \quad \text { and } \quad \operatorname{Curl} X_{n} \rightarrow C
$$

Thus,

$$
\operatorname{sym} X_{n}=\operatorname{sym} q_{n}-\operatorname{sym}\left(q_{n}-X_{n}\right) \rightarrow B-\operatorname{sym} A=\operatorname{sym}(B-A) \text { and } \operatorname{Curl} X_{n} \rightarrow C .
$$


Hence, it follows from the inequality $(3.88)$ that $\left(X_{n}\right)$ is a Cauchy sequence in $\left(\mathrm{Q},\|\cdot\|_{\mathrm{H}(\mathrm{Curl} ; \Omega)}\right)$. Hence, there exists $X \in \mathrm{Q}$ such that

$$
X_{n} \rightarrow X \quad \text { and } \quad \operatorname{Curl} X_{n} \rightarrow \operatorname{Curl} X=C \text { in } L^{2}\left(\Omega, \mathbb{R}^{3 \times 3}\right) .
$$

Now $q_{n}=X_{n}+\left(q_{n}-X_{n}\right) \rightarrow X+A$ and $\operatorname{sym} q_{n} \rightarrow \operatorname{sym}(X+A)=B$. Therefore,

$$
\begin{aligned}
\left\|\left(q_{n}, X_{n}\right)-(X+A, X)\right\|_{*}^{2}= & \left\|\left(q_{n}-(X+A), X_{n}-X\right)\right\|_{*}^{2}=\left\|\left(q_{n}-X_{n}\right)-A\right\|_{L^{2}}^{2} \\
& +\left\|\operatorname{sym} q_{n}-\operatorname{sym}(X+A)\right\|_{L^{2}}^{2}+\left\|\operatorname{Curl} X_{n}-\operatorname{Curl} X\right\|_{L^{2}}^{2} \rightarrow 0 .
\end{aligned}
$$

So, the sequence $\left(q_{n}, X_{n}\right)$ converges to $(X+A, X)$ in $\left(\mathrm{P} \times \mathrm{Q},\|\cdot\|_{*}\right)$.

Since the two normed spaces $\left(\mathrm{P} \times \mathrm{Q},\|\cdot\|_{P \times Q}\right)$ and $\left(\mathrm{P} \times \mathrm{Q},\|\cdot\|_{*}\right)$ are Banach and the identity mapping

$$
\mathrm{Id}:\left(\mathrm{P} \times \mathrm{Q},\|\cdot\|_{P \times Q}\right) \rightarrow\left(\mathrm{P} \times \mathrm{Q},\|\cdot\|_{*}\right)
$$

is linear and continuous, then as a consequence of the open mapping theorem, we find that

$$
\mathrm{Id}:\left(\mathrm{P} \times \mathrm{Q},\|\cdot\|_{*}\right) \rightarrow\left(\mathrm{P} \times \mathrm{Q},\|\cdot\|_{P \times Q}\right)
$$

is also linear and continuous. Therefore, the two norms $\|\cdot\|_{*}$ and $\|\cdot\|_{P \times Q}$ are equivalent and this completes the proof of the lemma.

We are now in a position to prove that the bilinear form $\boldsymbol{a}$ in (3.84) is Z-coercive.

Lemma 3.2 There exists a positive constant $C$ such that $\boldsymbol{a}(z, z) \geq C\|z\|_{Z}^{2}$ for every $z \in \mathbf{Z}$.

Proof. Let $z=(v, q, X) \in \mathrm{Z}=\mathrm{V} \times \mathrm{P} \times \mathrm{Q}$

$$
\begin{aligned}
\boldsymbol{a}(z, z) \geq m_{0}\|\operatorname{sym}(\nabla v-q)\|_{L^{2}}^{2}(\operatorname{from}(2.3)) & +\mu H_{\chi}\|X-q\|_{L^{2}}^{2} \\
& +\mu L_{c}^{2}\|\operatorname{Curl} X\|_{L^{2}}^{2}+\mu k_{1}\|\operatorname{sym} q\|_{L^{2}}^{2} \\
=m_{0}\left[\|\operatorname{sym} \nabla v\|_{L^{2}}^{2}+\|\operatorname{sym} q\|_{2}^{2}-2\langle\operatorname{sym} \nabla v, \operatorname{sym} q\rangle\right]+\mu H_{\chi}\|X-q\|_{L^{2}}^{2} & +\mu L_{c}^{2}\|\operatorname{Curl} X\|_{L^{2}}^{2}+\mu k_{1}\|\operatorname{sym} q\|_{L^{2}}^{2} \\
\geq & m_{0}(1-\theta)\|\operatorname{sym} \nabla v\|_{L^{2}}^{2}+\left[m_{0}\left(1-\frac{1}{\theta}\right)+\mu k_{1}\right]\|\operatorname{sym} q\|_{2}^{2}+\mu H_{\chi}\|X-q\|_{L^{2}}^{2} \\
& +\mu L_{c}^{2}\|\operatorname{Curl} X\|_{L^{2}}^{2} .
\end{aligned}
$$

Now, since the hardening constant $k_{1}>0$, we choose $\theta$ such that

$$
\frac{m_{0}}{m_{0}+\mu k_{1}}<\theta<1
$$

and using Korn's first inequality (see e.g. [62]) and Lemma 3.1, we then get two constants $C=C\left(\theta, m_{0}, \mu, H_{\chi}, k_{1}, L_{c}, \Omega\right)>0$ and $C^{\prime}=C^{\prime}\left(\theta, m_{0}, \mu, H_{\chi}, k_{1}, L_{c}, \Omega\right)>0$ such that

$$
\boldsymbol{a}(z, z) \geq C^{\prime}\left[\|\nabla v\|_{L^{2}}^{2}+\|(q, X)\|_{*}^{2}\right] \geq C\left[\|\nabla v\|_{L^{2}}^{2}+\|q\|_{L^{2}}^{2}+\|X\|_{H(\operatorname{Curl} ; \Omega)}^{2}\right]=C\|z\|_{Z}^{2} .
$$


Well-posedness for the microcurl model

\section{The microcurl model in polycrystalline gradient plasticity}

\subsection{The case with isotropic hardening}

The free-energy density $\Psi$ is given in the additively separated form

$$
\begin{aligned}
\Psi\left(\nabla u, \varepsilon_{p}, \mathcal{X}_{p}, \operatorname{Curl} \mathcal{X}_{p}, \eta_{p}\right):= & \underbrace{\Psi_{e}^{\operatorname{lin}}\left(\varepsilon_{e}\right)}_{\text {elastic energy }}+\underbrace{\Psi_{\text {micro }}^{\text {lin }}\left(\varepsilon_{p}, \mathcal{X}_{p}\right)}_{\text {micro energy }} \\
& +\underbrace{\Psi_{\text {curl }}^{\text {lin }}\left(\operatorname{Curl} \mathcal{X}_{p}\right)}_{\text {defect-like energy }(\mathrm{GND})} \underbrace{\Psi_{\text {iso }}^{\operatorname{lin}\left(\eta_{p}\right)}}_{\text {hardening energy (SSD) }},
\end{aligned}
$$

where

$$
\begin{aligned}
\Psi_{e}^{\operatorname{lin}}\left(\varepsilon_{e}\right) & :=\frac{1}{2}\left\langle\varepsilon_{e}, \mathbb{C}_{\text {iso }} \varepsilon_{e}\right\rangle=\frac{1}{2}\left\langle\operatorname{sym} \nabla u-\varepsilon_{p}, \mathbb{C}_{\text {iso }}\left(\operatorname{sym} \nabla u-\varepsilon_{p}\right)\right\rangle, \\
\Psi_{\text {micro }}^{\operatorname{lin}}\left(\varepsilon_{p}, \mathcal{X}_{p}\right) & :=\frac{1}{2} \mu H_{\chi}\left\|\varepsilon_{p}-\operatorname{sym} \mathcal{X}_{p}\right\|^{2}, \quad \Psi_{\text {curl }}^{\operatorname{lin}}\left(\operatorname{Curl} \mathcal{X}_{p}\right):=\frac{1}{2} \mu L_{c}^{2}\left\|\operatorname{Curl} \mathcal{X}_{p}\right\|^{2}, \\
\Psi_{\text {iso }}^{\operatorname{lin}}\left(\eta_{p}\right) & :=\frac{1}{2} \mu k_{2}\left|\eta_{p}\right|^{2} .
\end{aligned}
$$

Here, $\eta_{p}$ is the isotropic hardening variable.

It should be noted that there is no constraint on the skew-symmetric part of the microdeformation, skew $\mathcal{X}_{p}$, in (4.2), due to the fact that no plastic spin is considered in the original plasticity model for skew $\mathcal{X}_{p}$ to be compared with. It will be shown that, in spite of that, no indetermi-

nacy of skew $\mathcal{X}_{p}$ arises in the formulation ${ }^{5}$. This represents the most straightforward microcurl extension of a phenomenological polycrystal plasticity model.

\subsubsection{The balance equations.}

As in Section 3.2.1, we have the balance equations:

$$
\begin{aligned}
\operatorname{div} \sigma+f & =0 \\
\mu L_{c}^{2} \operatorname{Curl} \operatorname{Curl} \mathcal{X}_{p} & =-\mu H_{\chi}\left(\operatorname{sym} \mathcal{X}_{p}-\varepsilon_{p}\right) \in \operatorname{Sym}(3) \quad \text { (macroscopic bal }
\end{aligned}
$$

where (4.4) is supplemented by the boundary conditions

$$
\mathcal{X}_{p} \times\left. n\right|_{\Gamma_{\mathrm{D}}}=0 \quad \text { and } \quad\left(\operatorname{Curl} \mathcal{X}_{p}\right) \times\left. n\right|_{\partial \Omega \backslash \Gamma_{\mathrm{D}}}=0 .
$$

\subsubsection{The derivation of the dissipation inequality.}

The local free-energy imbalance states that

$$
\dot{\Psi}-\left\langle\sigma, \dot{\varepsilon}_{e}\right\rangle-\left\langle\sigma, \dot{\varepsilon}_{p}\right\rangle \leq 0 \text {. }
$$

Now we expand the first term, substitute (4.1) and get

$$
\left\langle\mathbb{C}_{\text {iso }} \varepsilon_{e}-\sigma, \dot{\varepsilon}_{e}\right\rangle-\left\langle\sigma, \dot{\varepsilon}_{p}\right\rangle-\mu H_{\chi}\left\langle\operatorname{sym} \mathcal{X}_{p}-\varepsilon_{p}, \dot{\varepsilon}_{p}\right\rangle+\mu k_{2} \eta_{p} \dot{\eta}_{p} \leq 0 .
$$

\footnotetext{
${ }^{5}$ No simple characterization of skew $\mathcal{X}_{p}$ for $H_{\chi} \rightarrow \infty$ is known at present.
} 
Since the inequality (4.7) must be satisfied for whatever elastic-plastic deformation mechanism, inlcuding purely elastic ones (for which $\dot{\eta}_{p}=0, \dot{\varepsilon}_{p}=0$ ), then it implies the infinitesimal stressstrain relation

$$
\sigma=\mathbb{C}_{\text {iso }} \varepsilon_{e}=2 \mu\left(\operatorname{sym} \nabla u-\varepsilon_{p}\right)+\lambda \operatorname{tr}\left(\operatorname{sym} \nabla u-\varepsilon_{p}\right) \mathbb{1}
$$

and the local reduced dissipation inequality

$$
-\left\langle\sigma, \dot{\varepsilon}_{p}\right\rangle-\mu H_{\chi}\left\langle\operatorname{sym} \mathcal{X}_{p}-\varepsilon_{p}, \dot{\varepsilon}_{p}\right\rangle+\mu k_{2} \eta_{p} \dot{\eta}_{p} \leq 0
$$

That is,

$$
\left\langle\sigma+\mu H_{\chi}\left(\operatorname{sym} \mathcal{X}_{p}-\varepsilon_{p}\right), \dot{\varepsilon}_{p}\right\rangle-\mu k_{2} \eta_{p} \dot{\eta}_{p} \geq 0,
$$

which can also be written in compact form as

$$
\left\langle\Sigma_{p}, \dot{\Gamma}_{p}\right\rangle \geq 0
$$

where

$$
\Sigma_{p}:=\left(\Sigma_{E}, g\right) \quad \text { and } \quad \Gamma_{p}=\left(\varepsilon_{p}, \eta_{p}\right)
$$

with $\Sigma_{E}$ being a symmetric Eshelby-type stress tensor and $g$ being a thermodynamic force-type variable conjugate to $\dot{\eta}_{p}$ and defined as

$$
\begin{aligned}
\Sigma_{E} & :=\sigma+\mu H_{\chi}\left(\operatorname{sym} \mathcal{X}_{p}-\varepsilon_{p}\right)=\sigma-\mu L_{c}^{2} \operatorname{Curl} \operatorname{Curl} \mathcal{X}_{p} \\
g & :=-\mu k_{2} \eta_{p} .
\end{aligned}
$$

\subsubsection{The flow rule}

We consider a yield function defined by

$$
\phi\left(\Sigma_{p}\right):=\left\|\operatorname{dev} \Sigma_{\mathrm{E}}\right\|+g-\sigma_{0} \quad \text { for } \Sigma_{p}=\left(\Sigma_{E}, g\right) .
$$

So the set of admissible (elastic) generalized stresses is defined as

$$
\mathcal{K}:=\left\{\Sigma_{p}=\left(\Sigma_{\mathrm{E}}, g\right) \mid \phi\left(\Sigma_{p}\right) \leq 0, g \leq 0\right\} .
$$

The principle of maximum dissipation gives the normality law

$$
\dot{\Gamma}_{p} \in N_{\mathcal{K}}\left(\Sigma_{p}\right) \text {, }
$$

where $N_{\mathcal{K}}\left(\Sigma_{p}\right)$ denotes the normal cone to $\mathcal{K}$ at $\Sigma_{p}$, which is the set of generalised strain rates $\dot{\Gamma}_{p}$ that satisfy

$$
\left\langle\bar{\Sigma}-\Sigma_{p}, \dot{\Gamma}_{p}\right\rangle \leq 0 \quad \text { for all } \bar{\Sigma} \in \mathcal{K} .
$$

Notice that $N_{\mathcal{K}}=\partial \chi_{\mathcal{K}}$ where $\chi_{\mathcal{K}}$ denotes the indicator function of the set $\mathcal{K}$ and $\partial \chi_{\mathcal{K}}$ denotes the subdifferential of the function $\chi_{\mathcal{K}}$.

Whenever the yield surface $\partial \mathcal{K}$ is smooth at $\Sigma_{p}$ then

$$
\dot{\Gamma}_{p} \in N_{\mathcal{K}}\left(\Sigma_{p}\right) \quad \Rightarrow \quad \exists \lambda \text { such that } \dot{\varepsilon}_{p}=\lambda \frac{\operatorname{dev} \Sigma_{\mathrm{E}}}{\left\|\operatorname{dev} \Sigma_{\mathrm{E}}\right\|} \quad \text { and } \quad \dot{\eta}_{p}=\lambda=\left\|\dot{\varepsilon}_{p}\right\|
$$


with the Karush-Kuhn Tucker conditions: $\lambda \geq 0, \phi\left(\Sigma_{p}\right) \leq 0$ and $\lambda \phi\left(\Sigma_{p}\right)=0$. Using convex analysis (Legendre-transformation) we find that
$\underbrace{\dot{\Gamma}_{p} \in \partial \chi_{\mathcal{K}}\left(\Sigma_{p}\right)}$
$\Leftrightarrow \quad \underbrace{\Sigma_{p} \in \partial \chi_{\mathcal{K}}^{*}\left(\dot{\Gamma}_{p}\right)}$
flow rule in its dual formulation
flow rule in its primal formulation

where $\chi_{\mathcal{K}}^{*}$ is the Fenchel-Legendre dual of the function $\chi_{\mathcal{K}}$ denoted in this context by $\mathcal{D}_{\text {iso }}$, the one-homogeneous dissipation function for rate-independent processes. That is, for every $\Gamma=(q, \beta)$,

$$
\begin{aligned}
\mathcal{D}_{\text {iso }}(\Gamma) & =\sup \left\{\left\langle\Sigma_{p}, \Gamma\right\rangle \mid \Sigma_{p} \in \mathcal{K}\right\} \\
& =\sup \left\{\left\langle\Sigma_{E}, q\right\rangle+g \beta \mid \phi\left(\Sigma_{E}, g\right) \leq 0, g \leq 0\right\} \\
& = \begin{cases}\sigma_{0}\|q\| & \text { if }\|q\| \leq \beta \\
\infty & \text { otherwise. }\end{cases}
\end{aligned}
$$

We get from the definition of the subdifferential $\left(\Sigma_{p} \in \partial \chi_{\mathcal{K}}^{*}\left(\dot{\eta}_{p}\right)\right)$ that,

$$
\mathcal{D}_{\text {iso }}(\Gamma) \geq \mathcal{D}_{\text {iso }}\left(\dot{\Gamma}_{p}\right)+\left\langle\Sigma_{p}, \Gamma-\dot{\Gamma}_{p}\right\rangle \text { for any } \Gamma \text {. }
$$

That is,

$$
\mathcal{D}_{\text {iso }}(q, \beta) \geq \mathcal{D}_{\text {iso }}\left(\dot{\varepsilon}_{p}, \dot{\eta}_{p}\right)+\left\langle\Sigma_{E}, q-\dot{\varepsilon}_{p}\right\rangle+g\left(\beta-\dot{\eta}_{p}\right) \quad \text { for any }(q, \beta)
$$

In the next sections, we present as in the case of signle-crystal gradient plasticity, a complete mathematical analysis of the model including both strong and weak fomrulations as well as a corresponding existence result.

\subsubsection{Strong formulation of the model}

To summarize, we have obtained the following strong formulation for the microcurl model in the poycrystalline infinitesimal gradient plasticity setting with isotropic hardening. Given $f \in$ $\mathrm{H}^{1}\left(0, T ; L^{2}\left(\Omega, \mathbb{R}^{3}\right)\right)$, the goal is to find:

(i) the displacement $u \in \mathrm{H}^{1}\left(0, T ; H_{0}^{1}\left(\Omega, \Gamma_{\mathrm{D}}, \mathbb{R}^{3}\right)\right)$,

(ii) the infinitesimal plastic strain $\varepsilon_{p} \in \mathrm{H}^{1}\left(0, T ; L^{2}(\Omega\right.$, $\left.\operatorname{Sym}(3) \cap \mathfrak{s l}(3))\right)$, the infinitesimal microdistortion $\mathcal{X}_{p}$ with sym $\mathcal{X}_{p} \in \mathrm{H}^{1}\left(0, T ; L^{2}(\Omega, \operatorname{Sym}(3) \cap \mathfrak{s l}(3))\right)$, Curl $\mathcal{X}_{p} \in \mathrm{H}^{1}\left(0, T ; L^{2}\left(\Omega, \mathbb{R}^{3 \times 3}\right)\right)$ and Curl Curl $\mathcal{X}_{p} \in \mathrm{H}^{1}\left(0, T ; L^{2}\left(\Omega, \mathbb{R}^{3 \times 3}\right)\right)$

such that the content of Table 4 holds.

\subsubsection{Weak formulation of the model}

Assume that the problem in Section 4.1.4 has a solution

$\left(u, \varepsilon_{p}, \mathcal{X}_{p}, \eta_{p}\right)$. Let $v \in H^{1}\left(\Omega, \mathbb{R}^{3}\right)$ with $v_{\mid \Gamma_{D}}=0$. Multiply the equilibrium equation with $v-\dot{u}$ and integrate in space by parts and use the symmetry of $\sigma$ and the elasticity relation to get

$$
\left.\int_{\Omega}\left\langle\mathbb{C}_{\text {iso }}\left(\operatorname{sym} \nabla u-\varepsilon_{p}\right)\right), \operatorname{sym} \nabla v-\operatorname{sym} \nabla \dot{u}\right\rangle d x=\int_{\Omega} f(v-\dot{u}) d x .
$$




\begin{tabular}{|c|c|}
\hline $\begin{array}{l}\text { Additive split of strain: } \\
\text { Equilibrium: } \\
\text { Microbalance: }\end{array}$ & $\begin{array}{l}\nabla u=e+p, \quad \varepsilon_{e}=\operatorname{sym} e, \quad \varepsilon_{p}=\operatorname{sym} p \\
\text { Div } \sigma+f=0 \text { with } \sigma=\mathbb{C}_{\text {iso }} \varepsilon^{e}=\mathbb{C}_{\text {iso }}\left(\operatorname{sym} \nabla u-\varepsilon_{p}\right) \\
\mu L_{c}^{2} \text { Curl Curl } \mathcal{X}_{p}=-\mu H_{\chi}\left(\operatorname{sym} \mathcal{X}_{p}-\varepsilon_{p}\right),\end{array}$ \\
\hline Free energy: & $\begin{aligned} \frac{1}{2}\left\langle\mathbb{C} . \varepsilon^{e}, \varepsilon^{e}\right\rangle+\frac{1}{2} \mu H_{\chi}\left\|\varepsilon_{p}-\operatorname{sym} \mathcal{X}_{p}\right\|^{2} & \\
& +\frac{1}{2} \mu L_{c}^{2}\left\|\operatorname{Curl} \mathcal{X}_{p}\right\|^{2}+\frac{1}{2} \mu k_{2}\left|\eta_{p}\right|^{2}\end{aligned}$ \\
\hline $\begin{array}{l}\text { Yield condition: } \\
\text { where }\end{array}$ & $\begin{array}{l}\phi\left(\Sigma_{E}\right):=\left\|\operatorname{dev} \Sigma_{E}\right\|+g-\sigma_{0} \leq 0 \\
\Sigma_{E}:=\sigma+\mu H_{\chi}\left(\operatorname{sym} \mathcal{X}_{p}-\varepsilon_{p}\right), \quad g=-\mu k_{2} \eta_{p}\end{array}$ \\
\hline Dissipation inequality: & $\left\langle\Sigma_{E}, \dot{\varepsilon}_{p}\right\rangle+g \dot{\eta}_{p} \geq 0$ \\
\hline Dissipation function: & $\mathcal{D}_{\text {iso }}(q, \beta):= \begin{cases}\sigma_{0}\|q\| & \text { if }\|q\| \leq \beta \\
\infty & \text { otherwise }\end{cases}$ \\
\hline Flow rule in primal form: & $\left(\Sigma_{E}, g\right) \in \partial \mathcal{D}_{\text {iso }}\left(\dot{\varepsilon}_{p}, \dot{\eta}_{p}\right)$ \\
\hline Flow rule in dual form: & $\dot{\varepsilon}_{p}=\lambda \frac{\operatorname{dev} \Sigma_{E}}{\left\|\operatorname{dev} \Sigma_{E}\right\|}$ \\
\hline $\begin{array}{l}\text { KKT conditions: } \\
\text { Boundary conditions for } \mathcal{X}_{p}: \\
\text { Function space for } \mathcal{X}_{p}:\end{array}$ & $\begin{array}{l}\lambda \geq 0, \quad \phi\left(\Sigma_{E}, g\right) \leq 0, \quad \lambda \phi\left(\Sigma_{E}, g\right)=0 \\
\mathcal{X}_{p} \times n=0 \text { on } \Gamma_{\mathrm{D}}, \quad\left(\operatorname{Curl} \mathcal{X}_{p}\right) \times n=0 \text { on } \partial \Omega \backslash \Gamma_{\mathrm{D}} \\
\mathcal{X}_{p}(t, \cdot) \in \mathrm{H}\left(\operatorname{Curl} ; \Omega, \mathbb{R}^{3 \times 3}\right)\end{array}$ \\
\hline
\end{tabular}

Table 4: The microcurl model in polycrystalline gradient plasticity with isotropic hardening. The boundary condition on $\mathcal{X}_{p}$ necessitates at least $\mathcal{X}_{p} \in \mathrm{H}\left(\mathrm{Curl} ; \Omega, \mathbb{R}^{3 \times 3}\right)$. This is proven to be the case in the next sections through a weak formulation of the model as a variational inequality.

Now, for any $X \in C^{\infty}(\bar{\Omega}, \mathfrak{s l}(3))$ such that $X \times n=0$ on $\Gamma_{\mathrm{D}}$ we integrate (4.4) over $\Omega$, integrate by parts the term with Curl Curl using the boundary conditions

$$
\left(X-\dot{\mathcal{X}}_{p}\right) \times n=0 \text { on } \Gamma_{\mathrm{D}}, \quad \operatorname{Curl} \mathcal{X}_{p} \times n=0 \text { on } \partial \Omega \backslash \Gamma_{\mathrm{D}}
$$

and get

$$
\int_{\Omega}\left[\mu L_{c}^{2}\left\langle\operatorname{Curl} \mathcal{X}_{p}, \operatorname{Curl} X-\operatorname{Curl} \dot{\mathcal{X}}_{p}\right\rangle-\mu H_{\chi}\left\langle\varepsilon_{p}-\operatorname{sym} \mathcal{X}_{p}, \operatorname{sym} X-\operatorname{sym} \dot{\mathcal{X}}_{p}\right\rangle\right] d x=0 .
$$

Moreover, for any $q \in C^{\infty}(\bar{\Omega}, \mathfrak{s l}(3))$ and any $\beta \in L^{2}(\Omega)$, we integrate (4.22) over $\Omega$ and get

$$
\begin{gathered}
\int_{\Omega} \mathcal{D}_{\text {iso }}(q, \beta) d x-\int_{\Omega} \mathcal{D}_{\text {iso }}\left(\dot{\varepsilon}_{p}, \dot{\eta}_{p}\right) d x-\int_{\Omega}\left\langle\mathbb{C}_{\text {iso }}\left(\operatorname{sym}(\nabla u)-\varepsilon_{p}\right), q-\dot{\varepsilon}_{p}\right\rangle d x \\
+\int_{\Omega}\left[\mu H_{\chi}\left\langle\varepsilon_{p}-\operatorname{sym} \mathcal{X}_{p}, q-\dot{\varepsilon}_{p}\right\rangle+\mu \alpha_{1} \eta_{p}\left(\beta-\dot{\eta}_{p}\right)\right] d x \geq 0 .
\end{gathered}
$$

Now adding up (4.23), (4.24) and (4.25) we get the following weak formulation of the problem 
in Section 4.1.4 in the form of a variational inequality:

$$
\begin{aligned}
\int_{\Omega}\left[\left\langle\mathbb{C}_{\text {iso }}(\right.\right. & \left.\left.\operatorname{sym} \nabla u-\varepsilon_{p}\right),(\operatorname{sym} \nabla v-q)-\left(\operatorname{sym} \nabla \dot{u}-\dot{\varepsilon}_{p}\right)\right\rangle+\mu L_{c}^{2}\left\langle\operatorname{Curl} \mathcal{X}_{p}, \operatorname{Curl} X-\operatorname{Curl} \dot{\mathcal{X}}_{p}\right\rangle \\
& \left.+\mu H_{\chi}\left\langle\operatorname{sym} \mathcal{X}_{p}-\varepsilon_{p},(\operatorname{sym} X-q)-\left(\operatorname{sym} \dot{\mathcal{X}}_{p}-\dot{\varepsilon}_{p}\right)\right\rangle+\mu k_{2} \eta_{p}\left(\beta-\dot{\eta}_{p}\right)\right] d x \\
& +\int_{\Omega} \mathcal{D}_{\text {iso }}(q, \beta) d x-\int_{\Omega} \mathcal{D}_{\text {iso }}\left(\dot{\varepsilon}_{p}, \dot{\eta}_{p}\right) d x \geq \int_{\Omega} f(v-\dot{u}) d x
\end{aligned}
$$

\subsubsection{Existence result for the weak formulation}

As in the case of single-crystal gradient plasticity in Section 3.2.6, the existence result for the weak formulation (4.26) is obtaned through the abstract machinery developed in [48] for mathematical problems in geometrically linear classical plasticity. To this aim, (4.26) is written as the variational inequality of the second kind: find $w=\left(u, \varepsilon_{p}, \mathcal{X}_{p}, \eta_{p}\right) \in \mathrm{H}^{1}(0, T ; Z)$ such that $w(0)=0, \dot{w}(t) \in W$ for a.e. $t \in[0, T]$ and

$$
\boldsymbol{a}(\dot{w}, z-w)+j(z)-j(\dot{w}) \geq\langle\ell, z-\dot{w}\rangle \text { for every } z \in W \text { and for a.e. } t \in[0, T],
$$

where $Z$ is a suitable Hilbert space and $W$ is some closed, convex subset of $Z$ to be constructed later,

$$
\begin{aligned}
\boldsymbol{a}(w, z)= & \int_{\Omega}\left[\left\langle\mathbb{C}_{\text {iso }}\left(\operatorname{sym} \nabla u-\varepsilon_{p}\right), \operatorname{sym} \nabla v-q\right\rangle+\mu L_{c}^{2}\left\langle\operatorname{Curl} \mathcal{X}_{p}, \operatorname{Curl} X\right\rangle\right. \\
& \left.+\mu H_{\chi}\left\langle\operatorname{sym} \mathcal{X}_{p}-\varepsilon_{p}, \operatorname{sym} X-q\right\rangle+\mu k_{2} \eta_{p} \beta\right] d x, \\
j(z)= & \int_{\Omega} \mathcal{D}_{\text {iso }}(q, \beta) d x, \\
\langle\ell, z\rangle= & \int_{\Omega} f v d x,
\end{aligned}
$$

for $w=\left(u, \varepsilon_{p}, \mathcal{X}_{p}, \eta_{p}\right)$ and $z=(v, q, X, \beta)$ in $\mathbf{Z}$.

The Hilbert space $\mathbf{Z}$ and the closed convex subset $\mathbf{W}$ are constructed in such a way that the functionals $\boldsymbol{a}, j$ and $\ell$ satisfy the assumptions in the abstract result in [48, Theorem 6.19]. The key issue here is the coercivity of the bilinear form $\boldsymbol{a}$ on the set $\mathrm{W}$, that is, $a(z, z) \geq C\|z\|_{Z}^{2}$ for every $z \in \mathrm{Z}$ and for some $C>0$.

We let

$$
\begin{aligned}
\mathrm{V} & :=\mathrm{H}_{0}^{1}\left(\Omega, \Gamma_{\mathrm{D}}, \mathbb{R}^{3}\right)=\left\{v \in \mathrm{H}^{1}\left(\Omega, \mathbb{R}^{3}\right) \mid v_{\mid \Gamma_{\mathrm{D}}}=0\right\} \\
\mathrm{P} & :=\mathrm{L}^{2}(\Omega, \mathfrak{s l}(3) \cap \operatorname{Sym}(3)) \\
\mathrm{Q} & :=\mathrm{H}_{0}(\operatorname{Curl} ; \Omega, \Gamma, \mathfrak{s l}(3)) \\
\Lambda & :=L^{2}(\Omega) \\
\mathrm{Z} & :=\mathrm{V} \times \mathrm{P} \times \mathrm{Q} \times \Lambda \\
\mathrm{W} & :=\{z=(v, q, X, \beta) \in \mathrm{Z} \mid\|q\| \leq \beta\}
\end{aligned}
$$


and define the norms

$$
\begin{aligned}
& \|v\|_{V}:=\|\nabla v\|_{L^{2}}, \quad\|q\|_{P}=\|q\|_{L_{2}}, \quad\|X\|_{Q}:=\|X\|_{\mathrm{H}(\mathrm{Curl} ; \Omega)}, \\
& \|z\|_{Z}^{2}:=\|v\|_{V}^{2}+\|q\|_{L^{2}}^{2}+\|X\|_{Q}^{2}+\|\beta\|_{L^{2}}^{2} \quad \text { for } z=(v, q, X, \beta) \in \mathbf{Z} .
\end{aligned}
$$

Let us show that the bilinear form $\boldsymbol{a}$ is coercive on $\mathbf{W}$. Let therefore $z=(v, q, X, \beta) \in \mathbf{W}$.

$$
\begin{aligned}
& \boldsymbol{a}(z, z) \geq m_{0}\|\operatorname{sym} \nabla v-q\|_{L^{2}}^{2}(\operatorname{from}(2.3))+\mu H_{\chi}\|\operatorname{sym} X-q\|_{L^{2}}^{2} \\
& +\mu L_{c}^{2}\|\operatorname{Curl} X\|_{L^{2}}^{2}+\mu k_{2}\|\beta\|_{L^{2}}^{2} \\
& =m_{0}\left[\|\operatorname{sym} \nabla v\|_{L^{2}}^{2}+\|q\|_{L^{2}}^{2}-2\langle\operatorname{sym} \nabla v, q\rangle\right]+\mu H_{\chi}\left[\|\operatorname{sym} X\|_{L^{2}}^{2}+\|q\|_{L^{2}}^{2}\right. \\
& -2\langle\operatorname{sym} X, q\rangle]+\mu L_{c}^{2}\|\operatorname{Curl} X\|_{L^{2}}^{2}+\mu k_{2}\|\beta\|_{L^{2}}^{2} \\
& \geq m_{0}\left[\|\operatorname{sym} \nabla v\|_{L^{2}}^{2}+\|q\|_{L^{2}}^{2}-\theta\|\operatorname{sym} \nabla v\|_{L^{2}}^{2}-\frac{1}{\theta}\|q\|_{L^{2}}^{2}\right] \quad \text { (Young's inequality) } \\
& +\mu H_{\chi}\left[\|\operatorname{sym} X\|_{L^{2}}^{2}+\|q\|_{L^{2}}^{2}-\theta\|\operatorname{sym} X\|_{L^{2}}^{2}-\frac{1}{\theta}\|q\|_{L^{2}}^{2}\right] \text { (Young's inequality) } \\
& +\mu L_{c}^{2}\|\operatorname{Curl} X\|_{L^{2}}^{2}+\frac{1}{2} \mu k_{2}\|\beta\|_{L^{2}}^{2}+\frac{1}{2} \mu k_{2}\|q\|_{L^{2}}^{2} \quad(\text { using }\|q\| \leq \beta) \\
& =m_{0}(1-\theta)\|\operatorname{sym} \nabla v\|_{L^{2}}^{2}+\left[\left(m_{0}+\mu H_{\chi}\right)\left(1-\frac{1}{\theta}\right)+\frac{1}{2} \mu k_{2}\right]\|q\|_{L^{2}}^{2} \\
& +\mu H_{\chi}(1-\theta)\|\operatorname{sym} X\|_{L^{2}}^{2}+\mu L_{c}^{2}\|\operatorname{Curl} X\|_{L^{2}}^{2}+\frac{1}{2} \mu k_{2}\|\beta\|_{L^{2}}^{2} .
\end{aligned}
$$

So, since the hardening constant $k_{2}>0$, it is possible to choose $\theta$ such that

$$
\frac{m_{0}+\mu H_{\chi}}{m_{0}+\mu H_{\chi}+\frac{1}{2} \mu k_{2}}<\theta<1
$$

and using Korn's first inequality (see e.g. [62]) and the Korn-type inequality for incompatible tensor fields established in $[72,73,74,75]$, namely

$$
\|X\|_{L^{2}}^{2} \leq C\left(\|\operatorname{sym} X\|_{L^{2}}^{2}+\|\operatorname{Curl} X\|_{L^{2}}^{2}\right) \quad \forall X \in \mathrm{H}_{0}\left(\operatorname{Curl} ; \Omega, \Gamma_{\mathrm{D}}, \mathbb{R}^{3 \times 3}\right),
$$

there exists some constant $C\left(m_{0}, \mu, H_{\chi}, k_{2}, L_{c}, \Omega\right)>0$ such that

$$
a(z, z) \geq C\left[\|v\|_{V}^{2}+\|q\|_{L^{2}}^{2}+\|X\|_{\mathrm{H}(\mathrm{Curl} ; \Omega)}^{2}+\|\beta\|_{L^{2}}^{2}\right]=C\|z\|_{Z}^{2} \quad \forall z=(v, q, X, \beta) \in \mathrm{W} .
$$

This shows existence for our microcurl model in polycrystalline gradient plasticity with isotropic hardening.

Remark 4.1 Arguing as in Section 3.2.7, we get for any two solutions $\left(u_{i}, \varepsilon_{p_{i}}, \mathcal{X}_{p_{i}}, \eta_{p_{i}}\right)$ with $i=1,2$ of (4.26) that

$$
u_{1}=u_{2}, \quad \varepsilon_{p_{1}}=\varepsilon_{p_{2}}, \quad \eta_{p_{1}}=\eta_{p_{2}}, \quad \operatorname{sym} \mathcal{X}_{p_{1}}=\operatorname{sym} \mathcal{X}_{p_{2}}, \quad \operatorname{Curl} \mathcal{X}_{p_{1}}=\operatorname{Curl}_{p_{2}}
$$

Now, using the Korn-type inequality for incompatible tensor fields established in [72, 73, 74, 75] and applied to $\mathcal{X}_{p_{1}}-\mathcal{X}_{p_{2}}$, namely

$$
\left\|\mathcal{X}_{p_{1}}-\mathcal{X}_{p_{2}}\right\|_{L^{2}}^{2} \leq C\left(\left\|\operatorname{sym}\left(\mathcal{X}_{p_{1}}-\mathcal{X}_{p_{2}}\right)\right\|_{L^{2}}^{2}+\left\|\operatorname{Curl}\left(\mathcal{X}_{p_{1}}-\mathcal{X}_{p_{2}}\right)\right\|_{L^{2}}^{2}\right),
$$

we also get that $\mathcal{X}_{p_{1}}=\mathcal{X}_{p_{2}}$ and this show the uniqueness of the weak/strong solution. 


\subsection{The model with linear kinematical hardening}

Here we consider the model where the isotropic hardening has been replaced with linear kinematical hardening. Here the free-energy is given by

$$
\begin{aligned}
\Psi\left(\nabla u, \varepsilon_{p}, \mathcal{X}_{p}, \operatorname{Curl} \mathcal{X}_{p}\right):= & \underbrace{\Psi_{e}^{\operatorname{lin}}\left(\varepsilon_{e}\right)}_{\text {elastic energy }}+\underbrace{\Psi_{\text {micro }}^{\operatorname{lin}}\left(\varepsilon_{p}, \mathcal{X}_{p}\right)}_{\text {micro energy }} \\
& +\underbrace{\Psi_{\text {curl }}^{\operatorname{lin}}\left(\operatorname{Curl} \mathcal{X}_{p}\right)}_{\text {defect-like energy }(\mathrm{GND})} \underbrace{\Psi_{\text {kin }}^{\operatorname{lin}\left(\varepsilon_{p}\right)}}_{\text {hardening energy (SSD) }}
\end{aligned}
$$

where

$$
\begin{aligned}
\Psi_{e}^{\operatorname{lin}}\left(\varepsilon_{e}\right) & :=\frac{1}{2}\left\langle\varepsilon_{e}, \mathbb{C}_{\mathrm{iso}} \varepsilon_{e}\right\rangle=\frac{1}{2}\left\langle\operatorname{sym} \nabla u-\varepsilon_{p}, \mathbb{C}_{\mathrm{iso}}\left(\operatorname{sym} \nabla u-\varepsilon_{p}\right)\right\rangle, \\
\Psi_{\text {micro }}^{\operatorname{lin}}\left(\varepsilon_{p}, \mathcal{X}_{p}\right) & :=\frac{1}{2} \mu H_{\chi}\left\|\varepsilon_{p}-\operatorname{sym} \mathcal{X}_{p}\right\|^{2}, \\
\Psi_{\text {curl }}^{\operatorname{lin}}\left(\operatorname{Curl} \mathcal{X}_{p}\right) & :=\frac{1}{2} \mu L_{c}^{2}\left\|\operatorname{Curl} \mathcal{X}_{p}\right\|^{2}, \quad \Psi_{\text {kin }}^{\operatorname{lin}}\left(\varepsilon_{p}\right):=\frac{1}{2} \mu k_{1}\left\|\varepsilon_{p}\right\|^{2} .
\end{aligned}
$$

The strong formulation of the model is presented in Table 5 while the weak formulation reads as

$$
\begin{aligned}
\int_{\Omega}\left[\left\langle\mathbb{C}_{\text {iso }}(\right.\right. & \left.\left.\operatorname{sym} \nabla u-\varepsilon_{p}\right),(\operatorname{sym} \nabla v-q)-\left(\operatorname{sym} \nabla \dot{u}-\dot{\varepsilon}_{p}\right)\right\rangle+\mu L_{c}^{2}\left\langle\operatorname{Curl} \mathcal{X}_{p}, \operatorname{Curl} \mathrm{Q}-\operatorname{Curl} \dot{\mathcal{X}}_{p}\right\rangle \\
& \left.+\mu H_{\chi}\left\langle\operatorname{sym} \mathcal{X}_{p}-\varepsilon_{p},(\operatorname{sym} \mathrm{Q}-q)-\left(\operatorname{sym} \dot{\mathcal{X}}_{p}-\dot{\varepsilon}_{p}\right)\right\rangle+\mu k_{1}\left\langle\varepsilon_{p} q-\dot{\varepsilon}_{p}\right\rangle\right] d x \\
& +\int_{\Omega} \mathcal{D}_{\operatorname{kin}}(q) d x-\int_{\Omega} \mathcal{D}_{\text {kin }}\left(\dot{\varepsilon}_{p}\right) d x \geq \int_{\Omega} f(v-\dot{u}) d x
\end{aligned}
$$

That is, setting $\mathrm{Z}:=\mathrm{V} \times \mathrm{P} \times \mathrm{Q}$ with $\mathrm{V}$ and $\mathrm{Q}$ defined in (4.31)-(4.33), $\mathrm{P}=L^{2}(\Omega, \operatorname{Sym}(3) \cap \mathfrak{s t}(3))$ and their norms in (4.37), we get the problem of the form: Find $w=\left(u, \varepsilon_{p}, \mathcal{X}_{p}\right) \in \mathrm{H}^{1}(0, T ; Z)$ such that $w(0)=0$ and

$$
\boldsymbol{a}(\dot{w}, z-w)+j(z)-j(\dot{w}) \geq\langle\ell, z-\dot{w}\rangle \text { for every } z \in \mathbf{Z} \text { and for a.e. } t \in[0, T],
$$

where

$$
\begin{aligned}
\boldsymbol{a}(w, z)= & \int_{\Omega}\left[\left\langle\mathbb{C}_{\text {iso }}\left(\operatorname{sym} \nabla u-\varepsilon_{p}\right), \operatorname{sym} \nabla v-q\right\rangle+\mu L_{c}^{2}\left\langle\operatorname{Curl} \mathcal{X}_{p}, \operatorname{Curl} X\right\rangle\right. \\
& \left.+\mu H_{\chi}\left\langle\operatorname{sym} \mathcal{X}_{p}-\varepsilon_{p}, \operatorname{sym} X-q\right\rangle+\mu k_{1}\left\langle\varepsilon_{p}, q\right\rangle\right] d x, \\
j(z)= & \int_{\Omega} \mathcal{D}_{\text {kin }}(q) d x, \\
\langle\ell, z\rangle= & \int_{\Omega} f v d x,
\end{aligned}
$$

for $w=\left(u, \varepsilon_{p}, \mathcal{X}_{p}\right)$ and $z=(v, q, X)$ in Z.

The existence and uniqueness result for the problem in (4.45)-(4.48) is obtained from [48, Theorem 6.15] as the bilinear form $\boldsymbol{a}$ is Z-coercive (arguing as in (4.38). 


\begin{tabular}{|c|c|}
\hline $\begin{array}{l}\text { Additive split of strain: } \\
\text { Equilibrium: } \\
\text { Microbalance: }\end{array}$ & $\begin{array}{l}\nabla u=e+p, \quad \varepsilon_{e}=\operatorname{sym} e, \quad \varepsilon_{p}=\operatorname{sym} p \\
\operatorname{Div} \sigma+f=0 \text { with } \sigma=\mathbb{C}_{\text {iso }} \varepsilon_{e}=\mathbb{C}_{\text {iso }}\left(\operatorname{sym} \nabla u-\varepsilon_{p}\right) \\
\mu L_{c}^{2} \text { Curl Curl } \mathcal{X}_{p}=-\mu H_{\chi}\left(\operatorname{sym} \mathcal{X}_{p}-\varepsilon_{p}\right),\end{array}$ \\
\hline Free energy: & $\begin{aligned} \frac{1}{2}\left\langle\mathbb{C}_{\mathrm{iso}} \varepsilon_{e}\right. & \left., \varepsilon_{e}\right\rangle+\frac{1}{2} \mu H_{\chi}\left\|\varepsilon_{p}-\operatorname{sym} \mathcal{X}_{p}\right\|^{2} \\
& +\frac{1}{2} \mu L_{c}^{2}\left\|\operatorname{Curl} \mathcal{X}_{p}\right\|^{2}+\frac{1}{2} \mu k_{1}\|\operatorname{sym} p\|^{2}\end{aligned}$ \\
\hline $\begin{array}{l}\text { Yield condition: } \\
\text { where } \\
\text { with }\end{array}$ & $\begin{array}{l}\phi\left(\Sigma_{E}\right):=\left\|\operatorname{dev} \Sigma_{E}\right\|-\sigma_{0} \leq 0 \\
\Sigma_{E}:=\sigma+\Sigma_{\text {micro }}^{\operatorname{lin}}+\Sigma_{\text {kin }}^{\operatorname{lin}} \\
\Sigma_{\text {minco }}^{\operatorname{lin}}=\mu H_{\chi}\left(\operatorname{sym} \mathcal{X}_{p}-\varepsilon_{p}\right)=-\mu L_{c}^{2} \text { Curl Curl } \mathcal{X}_{p} \\
\Sigma_{\text {kin }}^{\operatorname{lin}}=-\mu k_{1} \varepsilon_{p}\end{array}$ \\
\hline $\begin{array}{l}\text { Dissipation inequality: } \\
\text { Dissipation function: } \\
\text { Flow law in primal form: }\end{array}$ & $\begin{array}{l}\int_{\Omega}\left\langle\Sigma_{E}, \dot{\varepsilon}_{p}\right\rangle d x \geq 0 \\
\mathcal{D}_{\text {kin }}(q):=\sigma_{0}\|q\| \\
\Sigma_{E} \in \partial \mathcal{D}_{\text {kin }}\left(\dot{\varepsilon}_{p}\right)\end{array}$ \\
\hline Flow law in dual form: & $\dot{\varepsilon}_{p}=\lambda \frac{\operatorname{dev} \Sigma_{E}}{\left\|\operatorname{dev} \Sigma_{E}\right\|}$ \\
\hline $\begin{array}{l}\text { KKT conditions: } \\
\text { Boundary conditions for } \mathcal{X}_{p}: \\
\text { Function space for } \mathcal{X}_{p}:\end{array}$ & $\begin{array}{l}\lambda \geq 0, \quad \phi\left(\Sigma_{E}\right) \leq 0, \quad \lambda \phi\left(\Sigma_{E}\right)=0 \\
\mathcal{X}_{p} \times n=0 \text { on } \Gamma_{\mathrm{D}}, \quad\left(\operatorname{Curl} \mathcal{X}_{p}\right) \times n=0 \text { on } \partial \Omega \backslash \Gamma_{\mathrm{D}} \\
\mathcal{X}_{p}(t, \cdot) \in \mathrm{H}\left(\operatorname{Curl} ; \Omega, \mathbb{R}^{3 \times 3}\right)\end{array}$ \\
\hline
\end{tabular}

Table 5: The microcurl model in polycrystalline gradient plasticity with kinematical hardening. Also in this model, the boundary condition on $\mathcal{X}_{p}$ necessitates at least $\mathcal{X}_{p} \in \mathrm{H}\left(\mathrm{Curl} ; \Omega, \mathbb{R}^{3 \times 3}\right)$. Unlike the model with isotropic hardening for which uniqueness is obtained through the strong formulation, here we have uniqueness of the weak solution straight from the fomulation as a varational inequality.

\section{The relaxed linear micromorphic continuum}

The relaxed micromorphic model is a very special subclass of the micromorphic model approach in which the extra dependence on gradients of the micro-distortion appears only through the Curl-operator. In the static and isotropic cases, the purely elastic model consists of a two-field minimization problem for the displacement $u: \Omega \subset \mathbb{R}^{3} \rightarrow \mathbb{R}^{3}$ and the non-symmetric microdistortion tensor $\mathcal{X}_{p}: \Omega \subset \mathbb{R}^{3} \rightarrow \mathbb{R}^{3 \times 3}$ so that for

$$
\begin{gathered}
\mathcal{E}\left(u, \mathcal{X}_{p}\right):=\int_{\Omega}\left[\mu_{e}\left\|\operatorname{sym}\left(\nabla u-\mathcal{X}_{p}\right)\right\|^{2}+\mu_{c}\left\|\operatorname{skew}\left(\nabla u-\mathcal{X}_{p}\right)\right\|^{2}+\frac{\lambda_{e}}{2}\left(\operatorname{tr}\left(\nabla u-\mathcal{X}_{p}\right)\right)^{2}\right. \\
\left.+\mu_{\text {micro }}\left\|\operatorname{sym} \mathcal{X}_{p}\right\|^{2}+\frac{\lambda_{\text {micro }}}{2}\left(\operatorname{tr}\left(\mathcal{X}_{p}\right)\right)^{2}+\mu_{e} \frac{L_{c}^{2}}{2}\left\|\operatorname{Curl} \mathcal{X}_{p}\right\|^{2}\right] d x \\
\mathcal{E}\left(u, \mathcal{X}_{p}\right) \rightarrow \min \text { w.r.t }\left(u, \mathcal{X}_{p}\right)
\end{gathered}
$$

subject to displacement boundary conditions $\left.u\right|_{\Gamma_{\mathrm{D}}}=0$ and the tangential boundary conditions $\mathcal{X}_{p} \times\left. n\right|_{\Gamma_{\mathrm{D}}}=0$ (equivalent to $\left.\mathcal{X}_{p} \cdot \tau\right|_{\Gamma_{\mathrm{D}}}=0$ for all vectors $\tau$ tangent to $\Gamma_{\mathrm{D}}$ ). Here, $\mu_{e}$ and $\lambda_{e}$ with

$$
\mu_{e}>0 \quad \text { and } \quad 2 \mu_{e}+3 \lambda_{e}>0
$$


are new elastic material constants which are not the Lamé constants of linear elasticity. Wellposedness results in statics and dynamics have been obtained in [69, 70], making crucial use of a recently established Korn's inequality for incompatible tensor fields [72, 73, 74, 75]. The parameter $\mu_{c} \geq 0$ is called the Cosserat couple modulus and may be set to zero in this model.

Regarding the relation to the polycrystalline microcurl model (4.42)-(4.43), we see that in (5.1) the minimization variable $\mathcal{X}_{p}$ is elastically coupled to the displacement gradient $\nabla u$ instead of being (penalty)-coupled to the plastic distortion $p$ in the microcurl model (4.42)-(4.43).

In the single crystal microcurl model, the equation for the micro-distortion can be obtained from the one-field minimization problem

$$
\int_{\Omega}\left[\frac{1}{2} \mu H_{\chi}\left\|p-\mathcal{X}_{p}\right\|^{2}+\frac{1}{2} \mu L_{c}^{2}\left\|\operatorname{Curl} \mathcal{X}_{p}\right\|^{2}\right] d x \rightarrow \min \mathcal{X}_{p}
$$

at given plastic distortion $p$.

Now, if we let $\mu_{e}, \mu_{c}, \lambda_{e} \rightarrow \infty\left(\mathcal{X}_{p} \rightarrow \nabla u\right)$ then the static model turns indeed into a linear elastic model

$$
\int_{\Omega}\left[\mu_{\infty}\|\operatorname{sym} \nabla u\|^{2}+\frac{\lambda_{\infty}}{2}(\operatorname{tr}(\nabla u))^{2}\right] d x \rightarrow \min u,
$$

where $\lambda_{\infty}, \mu_{\infty}$ can be determined analytically [10].

The formulation (5.1) in the dynamic case has a number of distinguishing features. As it turns out, the so-called metamaterials with band-gaps at certain frequency ranges can be qualitatively and quantatively described. For this, a nonzero Cosserat couple modulus $\mu_{c}>0$ is mandatory. Materials that do not show band-gaps must be modelled with $\mu_{c}=0$. Note that the formulation (5.1) contains as the special case $\mu_{\text {micro }}, \lambda_{\text {micro }} \rightarrow \infty$ the well-known infinitesimal Cosserat model in which the additional field $\mathcal{X}_{p}$ is restricted to be skew-symmetric (i.e., $\mathcal{X}_{p}$ is set as $A \in \mathfrak{s o}(3))$ and the elastic minimization problem reads

$$
\begin{aligned}
& \int_{\Omega}\left[\mu\|\operatorname{sym} \nabla u\|^{2}+\frac{\lambda}{2}(\operatorname{tr}(\nabla u))^{2}+\mu_{c}\|\operatorname{skew}(\nabla u-A)\|^{2}+\mu \frac{L_{c}^{2}}{2}\|\operatorname{Curl} A\|^{2}\right] d x \\
& \rightarrow \min (u, A),
\end{aligned}
$$

see e.g. [10]. The latter formulation has been coupled to perfect plasticity in an endevour to regularize ill-posedness of perfect plasticity, see e.g. [66, 67]

\section{Conclusion}

Examples of finite element computations based on the microcurl single crystal models can be found in [17] where polycrystalline microstructures are discetized in order to account for grain size effects on the local stress and lattice curvatures fields inside the grains and on the overall Hall-Petch effect. Orowan-type size effects were addressed for laminate microstructures in [93]. It remains to implement the polycrystalline formulation proposed in the present work and to compare its response to that of polycrystalline aggregates using the single crystal model. In that way the new material parameters could be identified from this multiscale analysis. This would 
also help to decide between the two possible penalty couplings, namely

$$
\underbrace{\frac{1}{2} \mu H_{\chi}\left\|p-\mathcal{X}_{p}\right\|^{2}}_{\text {direct coupling }} \quad \text { versus } \quad \underbrace{\frac{1}{2} \mu H_{\chi}\left\|\operatorname{sym}\left(p-\mathcal{X}_{p}\right)\right\|^{2}}_{\begin{array}{c}
\text { symmetric coupling } \\
\text { treated in this work }
\end{array}} .
$$

Mathematically, both formulations are well-posed, provided sufficient hardening is present. The direct coupling has the advantage of a clear penalty interpretation while the symmetric coupling does not see the plastic spin altogether, which may be advantageous from a modelling and implementational point of view.

The present mathematical analysis was performed within the infinitesimal framework. The reader is referred to [8] for a finite deformation formulation of the microcurl single crystal model that can be used for further applications involving significant lattice rotations and strains.

\section{References}

[1] E.C. Aifantis. On the microstructural origin of certain inelastic models. ASME J. Eng. Mater. Technol., 106:326-330, 1984.

[2] E.C. Aifantis. The physics of plastic deformation. Int. J. Plasticity, 3:211-247, 1987.

[3] E.C. Aifantis. On the role of gradients in the localization of deformation and fracture. Int. J. Engrg. Sci., 30:1279-1299, 1992.

[4] E.C. Aifantis. Update on a class of gradient theories. Mechanics of Materials, 35:259-280, 2003.

[5] H.D. Alber. Materials with Memory. Initial-Boundary Value Problems for Constitutive Equations with Internal Variables. volume 1682 of Lecture Notes in Mathematics. Springer, Berlin, 1998.

[6] L. Anand, O. Aslan, S.A. Chester. A large-deformation gradient theory for elastic-plastic materials: Strain softening and regularization pf shear bands. Int. J. Plasticity, 30-31:116-143, 2012.

[7] L. Anand, M.E. Gurtin, B.D. Reddy. The stored energy of cold work, thermal annealing, and other thermodynamic issues in single crystal plasticity at small length scales. Int. J. Plasticity, 64:1-25, 2015.

[8] O. Aslan, N.M. Cordero, A. Gaubert, S. Forest. Micromorphic approach to single crystal plasticity and damage. Int. J. Engng. Sci., 49:1311-1325, 2011.

[9] L. Bardella, A. Panteghini. Modelling the torsion of thin metal wires by distortion gradient plasticity. J. Mech. Phys. Solids. 78:467-492, 2015.

[10] G. Barbagallo, M.V. D'Agostino, R. Abreu, I.-D. Ghiba, A. Madeo, P. Neff. Transparent anisotropy for the relaxed micromorphic model: macroscopic consistency conditions and long wave length asymptotics. http://arxiv.org/pdf/1601.03667v2.pdf.

[11] S. Bargmann, B.D. Reddy, B. Klusemann. A computational study of a model of single-crystal strain gradient viscoplasticity with a fully-interactive hardening relation. Int. J. Solids Structures. 51(15-16):2754-2764, 2014.

[12] S. Bauer, P. Neff, D. Pauly, G. Starke. New Poincaré-type inequalities, Comptes Rendus Math. 352(4):163$166,2014$.

[13] S. Bauer, P. Neff, D. Pauly, G. Starke. Dev-Div-and DevSym-devCurl-inequalities for incompatible square square tensor fields with mixed boundary conditions. ESAIM Control Optim. Calc. Var., 22(1):112-133, 2016.

[14] E. Bayerschen, T. Böhlke. Power-law defect energy in a single-crystal gradient plasticity framework: A computational study. http://arxiv.org/pdf/1507.060\%9v3.pdf.

[15] H.-J. Chang, A. Gaubert, M. Firvel, S. Berbenni, O. Bouaziz, S. Forest. Analysis of particle induced dislocation structures using three-dimensional dislocation dynamics and strain gradient plasticity, Comput. Mater. Sci.. 52:33-39, 2012. 
[16] M. Chiricotto, L. Giacomelli, G. Tomassetti. Dissipative scale effects in strain-gradient plasticity: the case of simple shear. http://arxiv.org/pdf/1501.05668.pdf.

[17] N.M. Cordero, S. Forest, E.P. Busso. Micromorphic modelling of grain size effects in polycrystals, GAMMMitt. 36(2):186-202, 2013.

[18] N.M Cordero, A. Gaubert, S. Forest, E.P. Busso, F. Gallerneau, S. Kruch. Size effects in generalised continuum crystal plasticity for two-phase laminates. J. Mech. Phys. Solids. 58:1963-1994, 2010.

[19] R. De Wit. A view of the relation between the continuum theory of lattice defects and non-Euclidean geometry in the linear approximation. Int. J. Engng. Sci., 19:1475-1506, 1981.

[20] J.K. Djoko, F. Ebobisse, A.T. McBride, B.D. Reddy. A discontinuous Galerkin formulation for classical and gradient plasticity. Part 1: Formulation and analysis. Comput. Methods Appl. Mech. Engrg.. 196:3881-3897, 2007.

[21] J.K. Djoko, F. Ebobisse, A.T. McBride, B.D. Reddy. A discontinuous Galerkin formulation for classical and gradient plasticity. Part 2: Algorithms and numerial analysis. Comput. Methods Appl. Mech. Engrg., 197:1-22, 2007.

[22] F. Ebobisse, K. Hackl, P. Neff. A canonical rate-independent model of geometrically linear isotropic gradient plasticity with isotropic hardening and plastic spin accounting for the Burgers vector. http://arxiv.org/pdf/1603.00271.pdf.

[23] F. Ebobisse, A.T. McBride, B.D. Reddy. On the mathematical formulations of a model of gradient plasticity, in ed. B.D. Reddy, IUTAM-Symposium on Theoretical, Modelling and Computational Aspects of Inelastic Media (in Cape Town, 2008). 117-128. Springer, Berlin, 2008.

[24] F. Ebobisse, P. Neff. Existence and uniqueness in rate-independent infinitesimal gradient plasticity with isotropic hardening and plastic spin. Math. Mech. Solids, 15:691-703, 2010.

[25] F. Ebobisse, P. Neff, B.D. Reddy. Existence results in dislocation based rate-independent isotropic gradient plasticity with kinematical hardening and plastic spin: The case with symmetric local backstress. http://arxiv.org/pdf/1504.01973.pdf.

[26] N.A. Fleck, J.W. Hutchinson. Strain gradient plasticity. Advances in applied mechanics, J.W. Hutchinson and T.Y. Wu (Eds), 33:295-361, 1997.

[27] N.A. Fleck, J.W. Hutchinson. A reformulation of strain gradient plasticity. J. Mech. Phys. Solids, 49:2245$2271,2001$.

[28] N.A. Fleck, J.R. Willis. A mathematical basis for strain-gradient plasticity theory. Part I: scalar plastic multiplier. J. Mech. Phys. Solids, 57:161-177, 2009.

[29] N.A. Fleck, J.R. Willis. A mathematical basis for strain-gradient plasticity theory. Part II: Tensorial plastic multiplier. J. Mech. Phys. Solids, 57:1045-1057, 2009.

[30] S. Forest. Modeling slip, kink and shear banding in classical and generalized single crystal plasticity. Acta Materialia, 46(9):3265-3281, 1998.

[31] S. Forest. Questioning size effects as predicted by strain gradient plasticity. J. Mech. Behav. Mater., 22(34):101-110, 2013.

[32] S. Forest. Nonlinear regularization operators as derived from the micromorphic approach to gradient elasticity, viscoplasticity and damage. Proc. R. Soc. A, 472:20150755, 2015.

[33] S. Forest, K. Ammar, B. Appolaire, N.M. Cordero, A. Gaubert. Micromorphic approach to crystal plasticity and phase transition. In J. Schroeder and K. Hackl, editors, Plasticity and beyond, 131-198. CISM International Centre for Mechanical Sciences, Courses and Lectures No. 550, Springer, 2014.

[34] S. Forest, R. Sievert. Nonlinear microstrain theories. Int. J. Solids Structures, 43:7224-7245, 2006.

[35] G. Francfort, A. Giacomini, J.J. Marigo. The elasto-plasticity exquisite corpse: A Suquet legacy. J. Mech. Phys. Solids, in press.

[36] P. Franciosi, A. Zaoui. Crystal hardening and the issue of uniqueness. Int. J. Plasticity, 7:295-311, 1991.

[37] I.-D. Ghiba, P. Neff, A. Madeo, I. Münch. A variant of the linear isotropic indeterminate couple stress model with symmetric local force-stress, symmetric nonlocal force-stress, symmetric couple-stresses and complete traction boundary conditions. http://arxiv.org/pdf/1504.00868v1.pdf, to appear in Math. Mech. Solids. 
[38] P. Gudmundson. A unified treatment of strain gradient plasticity. J. Mech. Phys. Solids, 52:1379-1406, 2004.

[39] M.E. Gurtin. A gradient theory of single-crystal visco-plasticity that accounts for geometrically necessary dislocations. J. Mech. Phys. Solids, 50:5-32, 2002.

[40] M.E. Gurtin. A gradient theory of small deformation isotropic plasticity that accounts for the Burgers vector and for dissipation due to plastic spin. J. Mech. Phys. Solids, 52:2545-2568, 2004.

[41] M.E. Gurtin, L. Anand. A theory of strain gradient plasticity for isotropic, plastically irrotational materials. Part I: Small deformations. J. Mech. Phys. Solids, 53:1624-1649, 2005.

[42] M.E. Gurtin, L. Anand. Thermodynamics applied to gradient theories involving the accumulated plastic strain: The theories of Aifantis and Fleck and Hutchinson and their generalization. J. Mech. Phys. Solids, 57:405-421, 2009.

[43] M.E. Gurtin, E. Fried, L. Anand. The Mechanics and Thermodynamics of Continua. Cambridge University Press, Cambridge, 2010.

[44] M.E. Gurtin, A. Needleman. Boundary conditions in small-deformation, single-crystal plasticity that account for the Burgers vector. J. Mech. Phys. Solids. 53:1-31, 2005.

[45] M.E. Gurtin, B.D. Reddy. Gradient single-crystal plasticity within a von Mises-Hill framework based on a new formulation of self- and latent-hardening relations. J. Mech. Phys. Solids. 68:134-160, 2014.

[46] K. Hackl, Generalized standard media and variational principles in classical and finite strain elastoplasticity. J. Mech. Phys. Solids, 45:667-688, 1997.

[47] K. Hackl, F.D. Fischer. On the relation between the principle of maximum dissipation and inelastic evolution given by dissipation potentials. Proc. Roy. Soc. A, 464:117-132, 2008.

[48] W. Han, B.D. Reddy. Plasticity: Mathematical Theory and Numerical Analysis. 2nd edition, Springer-Verlag, New-York, 2013.

[49] R. Hill. The Mathematical Theory of Plasticity. Oxford University Press, New York, 1950.

[50] D.M. Kochmann, K. Hackl. The evolution of laminates in finite crystal plasticity: a variational approach. Cont. Mech. Thermod., 23:63-85, 2011.

[51] N. Kraynyukova, P. Neff, S. Nesenenko, K. Chełmiński. Well-posedness for dislocation based gradient viscoplasticity with isotropic hardening. http://arxiv.org/pdf/1411.1295v1.pdf, to appear in Nonlinear Analysis Series B: Real World Applications.

[52] E. Kröner. Continuum theory of defects. In: Les Houches, Session 35, 1980 - Physique des defauts, R. Balian et al. (Eds.). North-Holland, New York, pp. 215-315, 1981.

[53] J. Lubliner. Plasticity Theory. Dover Publications, Mineola, New York, 2008.

[54] M. Lucchesi, M. Šilhavý. Il'yushin's conditions in non-isothermal plasticity. Arch. Ration. Mech. Anal.. 113:121-163, 1991.

[55] A. Mainik, A. Mielke. Existence results for energetic models for rate-independent systems. Calc. Var. Partial. Differ. Equ., 22(1):72-99, 2005.

[56] M. Menzel, P. Steinmann. On the formulation of higher gradient plasticity for single and polycrystals. J. Phys. France, 8:239-247, 1998.

[57] M. Menzel, P. Steinmann. On the continuum formulation of higher gradient plasticity for single and polycrystals. J. Mech. Phys. Solids, 48:1777-1796, 2000. Erratum: 49:1179-1180, 2001.

[58] S.D. Mesarovic, S. Forest, J.P. Jaric. Size-dependent energy in crystal plasticity and continuum dislocation models. Proc. R. Soc. A, 471:20140868, 2015.

[59] A. Mielke. Analysis of energetic models for rate-independent materials. In T. Li, editor, Proceedings of the Int. Congress of Mathematicians 2002, Beijing, III: 817-828. Higher Education Press, 2002.

[60] A Mielke. Evolution of rate-independent systems. In. A. Dafermos and E. Feireisl, editors, Evolution equations. Vol. II, Handb. Differ. Equ., pages 461-559. Elsevier/North-Holland, Amsterdam, 2005.

[61] H.B. Mühlhaus, E.C. Aifantis. A variational principle for gradient plasticity. Int. J. Solids Struct. 28(7):845853, 1991.

[62] P. Neff. On Korn's first inequality with non-constant coefficients Proc. Roy. Soc. Edinburgh Sect. A. 132(1):221-243, 2002. 
[63] P. Neff. Existence of minimizers for a finite-strain micromorphic elastic solid. Proc. R. Soc. Edinb. A 136:9971012, 2006.

[64] P. Neff. Remarks on invariant modelling in finite strain gradient plasticity. Technische Mechanik, 28(1):13-21, 2008.

[65] P. Neff. Uniqueness of strong solutions in infinitesimal perfect gradient plasticity. In ed. B.D. Reddy, IUTAMSymposium on Theoretical, Modelling and Computational Aspects of Inelastic Media (in Cape Town, 2008). 129-140. Springer, Berlin, 2008.

[66] P. Neff, K. Chełmiński. Well-posedness of dynamic Cosserat plasticity. Appl. Math. Optimization, 56(1):19-35, 2007.

[67] P. Neff, K. Chełmiński, W. Müller, C. Wieners. A numerical solution methof for an infinitesimal elastic-plastic Cosserat model. Math. Mod. Meth. Appl. Sci. (M3AS), 17(8):1-29, 2007.

[68] P. Neff, K. Chełmiński, H.D. Alber. Notes on strain gradient plasticity. Finite strain covariant modelling and global existence in the infinitesimal rate-independent case. Math. Mod. Meth. Appl. Sci., 19(2):1-40, 2009.

[69] P. Neff, I.-D. Ghiba, A. Madeo, L. Placidi, G. Rosi. A unifying perspective: the relaxed linear micromorphic continuum. Cont. Mech. Therm., 26:639-681, 2014.

[70] P. Neff, I.-D. Ghiba, M. Lazar, A. Madeo. The relaxed linear micromorphic continuum: well-posedness of the static problem and relations to the gauge theory of dislocations. Quart. J. Mech. Appl. Math.. 68:53-84, 2015.

[71] P. Neff, I. Münch. Curl bounds Grad on SO(3). ESAIM Control Optim. Calc. Var. 14(1):148-159, 2008.

[72] P. Neff, D. Pauly, K.J. Witsch. On a canonical extension of Korn's first and Poincaré's inequalities to H(Curl) motivated by gradient plasticity with plastic spin. Comp. Rend. Math. 349(23-24):1251-1254, 2011.

[73] P. Neff, D. Pauly, K.J. Witsch. On a canonical extension of Korn's first and Poincaré's inequalities to H(Curl). J. Math. Sci. (NY), 185(5):721-727, 2012.

[74] P. Neff, D. Pauly, K.J. Witsch. Maxwell meets Korn: A new coercive inequality for tensor fields with square integrable exterior derivatives. Math. Methods Applied Sciences. 35(1):65-71, 2012.

[75] P. Neff, D. Pauly, K.J. Witsch. Poincaré meets Korn via Maxwell: Extending Korn's first inequality to incompatible tensor fields. J. Diff. Equations. 258(4):1267-1302, 2014.

[76] P. Neff, A. Sydow, C. Wieners. Numerical approximation of incremental infinitesimal gradient plasticity. Int. J. Num. Meth. Engrg. 77(3):414-436, 2009.

[77] S. Nesenenko, P. Neff. Well-posedness for dislocation based gradient visco-plasticity I: Subdifferential case. SIAM J. Math. Anal.. 44(3):1695-1712, 2012.

[78] S. Nesenenko, P. Neff. Well-posedness for dislocation based gradient visco-plasticity II: General nonassociative monotone plastic flow. Math. Mech. Complex Systems. 1(2):149-176, 2013.

[79] Q.-S. Nguyen. Variational principles in the theory of gradient plasticity. C. R. Mecanique. 339:743-750, 2011.

[80] J.F. Nye. Some geometrical relations in dislocated solids. Acta Metall.. 1:153-162, 1953.

[81] M. Ortiz, E. Repetto. Nonconvex energy minimization and dislocation structures in ductile single crystals. J. Mech. Phys. Solids, 47(2):397-462, 1999.

[82] L.H. Poh. Scale transition of a higher order plasticity model - A consistent homogenization theory from meso to macro. J. Mech. Phys. Solids. 61:2692-2710, 2013.

[83] L.H. Poh, R.H.J. Peerlings. The plastic rotation effect in an isotropic gradient plasticity model for applications at the meso scale. Int. J. Solids Struct., 78-79:57-69, 2016.

[84] B.D. Reddy. The role of dissipation and defect energy in variational formulations of problems in straingradient plasticity. Part 1: Polycrystalline plasticity. Cont. Mech. Therm.. 23:527-549, 2011.

[85] B.D. Reddy. The role of dissipation and defect energy in variational formulations of problems in straingradient plasticity. Part 2: Single-crystal plasticity. Cont. Mech. Therm., 23:551-572, 2011.

[86] B.D. Reddy, F. Ebobisse, A. McBride. Well-posedness of a model of strain gradient plasticity for plastically irrotational materials. Int. J. Plasticity, 24:55-73, 2008.

[87] B.D. Reddy, C. Wieners, B. Wohlmuth. Finite element analysis and algorithms for single-crystal straingradient plasticity. Int. J. Num. Meth. Engr. 90:784-804, 2014. 
[88] M.B. Rubin. Physical reasons for abandoning plastic deformation measures in plasticity and visco-plasticity. Arch. Mech., 53:519-539, 2001.

[89] J.C. Simo, T.J.R. Hughes. Computational inelasticity. Singer-Verlag, New York, 1998.

[90] B. Svendsen. Continuum thermodynamic models for crystal plasticity including the effects of geometrically necessary dislocations. J. Mech. Phys. Solids. 50(25):1297-1329, 2002.

[91] B. Svendsen, P. Neff, A. Menzel. On constitutive and configurational aspects of models for gradient continua with microstructure. Z. Angew. Math. Mech., 89(8):687-697, 2009.

[92] R.L.J.M. Ubachs, P.J.G. Schreurs, M.G.D. Geers. A nonlocal diffuse interface model for microstructure evolution of tin-lead splder. J. Mech. Phys. Solids. 52:1763-1792, 2004.

[93] S. Wulfinghof, S. Forest, T. Böhlke. Strain gradient plasticity modeling of the cyclic behavior of laminate microstructures. J. Mech. Phys. Solids. 79:1-20, 2015. 